\title{
Holger Helbig
}

\section{Reduktion und Rhetorik}

\section{Über die Bedeutung von Dingen in Ausstellungen}

\begin{abstract}
Ein und dasselbe Ding kann als Exponat in verschiedenen Ausstellungen verschiedene Bedeutungen annehmen: Mithilfe ein und desselben Dings lassen sich ganz unterschiedliche Themen darstellen oder Aussagen machen. Was ein Exponat in einer Ausstellung bedeutet, hängt wesentlich von zwei Kontexten ab: von dem, aus dem es als Ding entnommen wird, und von dem, in den es als Exponat gesetzt wird. Verallgemeinernd lässt sich sagen, dass die Inszenierung in der Ausstellung den ursprünglichen Kontext reduziert und den neuen Kontext rhetorisch auflädt. Von dieser Annahme ausgehend wird ein Beschreibungsmodell für die Strategien musealer Präsentation entwickelt und am Beispiel eines Ananaspräparats in zwei Ausstellungen erprobt.
\end{abstract}

Das teure Actionkino hat ein Faible für Museen. Das liegt zum einen am gattungstypischen Glanz der Lokalität und zum anderen am Flair der Hochkultur. Im Falle von Museen ist die Verbindung von Bildung und Unterhaltung akzeptiert, im Falle von Action nicht. Dass Bildung unterhaltend sein kann, war zuletzt in Skyfall (2012) zu besichtigen.

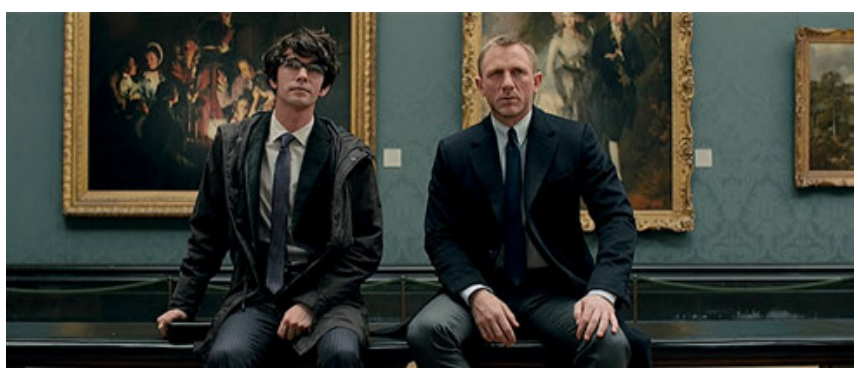

Abb. 1: Skyfall (2012): Bond und Q. in der National Gallery, London (Filmstill).

Ein alternder, angeschlagener Agent, Bond, James Bond mit Namen, und ein aufstrebender, pragmatisch veranlagter Nerd, Q., wie Quartiermaster, sind gezwungen, sich an einem unverdächtigen Ort in der Londoner Öffentlichkeit zu treffen. Man entscheidet sich für die National Gallery. Bond wartet unauffällig auf einer Bank vor William Turners The „Fighting Temeraire“ tugged to her last Berth to be 


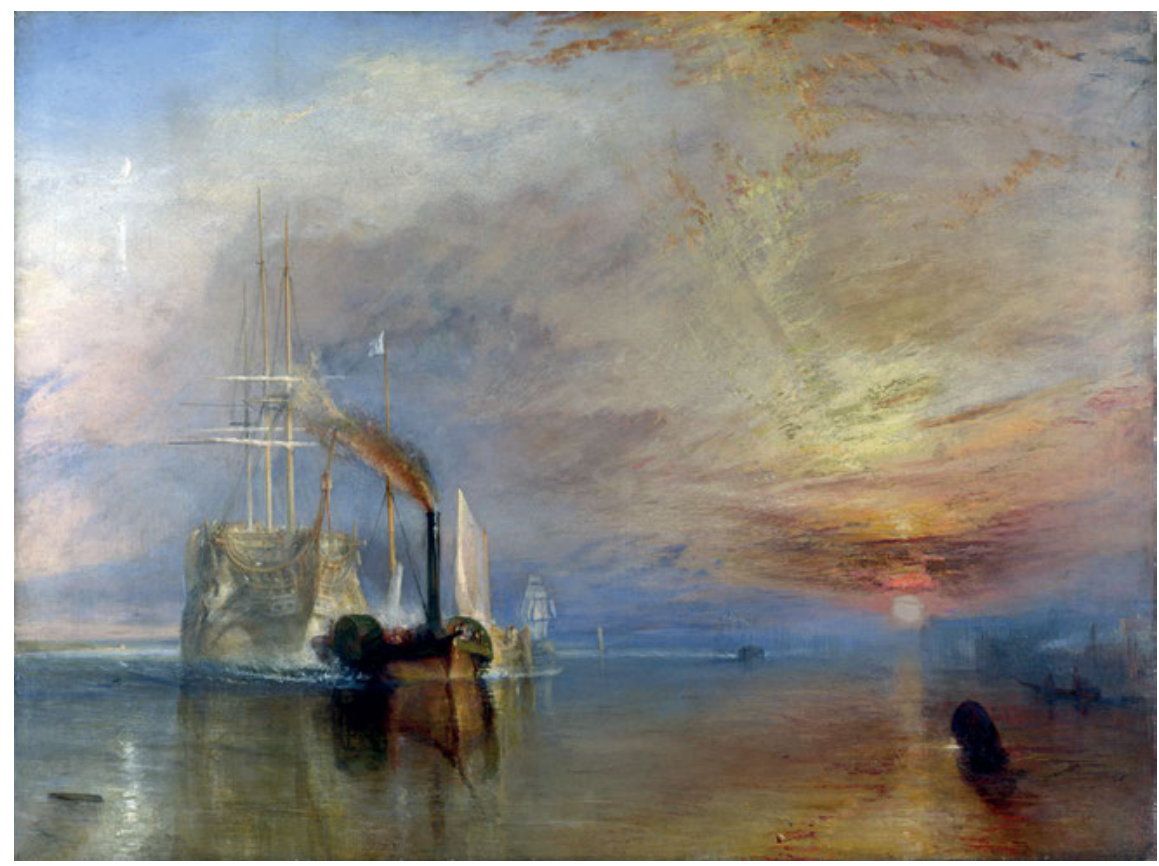

Abb. 2: William Turner: The „Fighting Temeraire“ tugged to her last Berth to be broken up (1839).

broken up (1839). Der Generationen jüngere Geheimdienstler nimmt neben ihm Platz und betrachtet das Bild aufmerksam. Die Kollegen kommen in Kontakt:

Q.: Always makes me feel a little melancholy. A grand old warship being ignominiously hauled away for scrap. - The inevitability of time, don't you think? What do you see?

Bond: A bloody big ship. ${ }^{1}$

Der empirisch grundierte Realismus, den Bond hier zur Schau stellt, hat in den letzten Jahren auch in den Museum Studies um sich gegriffen. Etliche akademische Annahmen über Sinn und Zweck von Museen sind als gutgemeinte, aber kaum haltbare Vorurteile verabschiedet worden. ${ }^{2}$ An die Stelle der Gewissheiten ist eine erfrischende Unübersichtlichkeit getreten. In der aktuellen Enzyklopädie

1 James Bond: Skyfall (Regie: Sam Mendes, USA/GB 2012), Szene 9. - Für Zu- und Einspruch danke ich Stefanie Kohl, Udo Andraschke und Andreas Lorenczuk.

2 Für einen instruktiven Überblick über die Veränderungen vgl. Stephen E. Weil: From Being About Something to Being for Somebody. In: ders.: Making Museums Matter. Washington, DC: Smithsonian, 2002. S. 28-52. Weil zitiert mit dem Titel Joanne Clever: Doing Children's Museums. Charlotte, VT: Williamson Publishing, 1992. S. 9. 
der Museum Studies heißt es - ohne Bezug zu Bond - über die Besucher von Kunstausstellungen:

[W]e can only assume that people go to see things that are there. Some people are interested to learn about those things - although it is impossible to know what - while others prefer to be left alone, to have an „,aesthetic experience“, or to see things ,as they are“. But since things are not, in any simple sense, since they only exist within the frame of a circumscribed materiality and institutionality, it seems pointless to subject museum work to this illusion. ${ }^{3}$ (Herv. im Orig.)

Dieser nüchterne Befund über die Möglichkeiten des Museums greift auf einen zentralen Ansatz der Museums-Forschung und sein wichtigstes Ergebnis zurück. Der Ansatz ist jener, der bei den Überlegungen zur Funktionsweise der Institution das einzelne Ding in den Mittelpunkt stellt. Er hat in der Folge des material turn zu Schlagworten wie ,Objekt-Geschichte،, ,Theorie der Dinge“ und der ebenso griffigen wie umstrittenen Phrase ,Things that talk' geführt, $\mathrm{zu}$ der das deutsche Pendant ,Sprache der Dinge“ gehört. ${ }^{4}$ Dass sich noch keine Nomenklatur der theoretischen Felder durchgesetzt hat und der Streit um die disziplinär adäquate Sprechweise erst am Anfang steht, macht einen guten Teil der angesprochenen Unübersichtlichkeit aus. ${ }^{5}$ Unumstritten ist aber die Einsicht, dass die Bedeutung eines Dings innerhalb des Museums nicht konstant ist. Vielmehr besteht ein Museum aus verschiedenen Räumen - etwa dem Depot und dem Ausstel-

3 Mieke Bal: Exposing the Public. In: A Companion to Museum Studies. Hrsg. von Sharon Macdonald. Malden, MA: Wiley-Blackwell, 2011. S. 525-542, hier S. 525.

4 Vgl. dazu Lorraine Daston: Speechless. In: Things That Talk: Object Lessons from Art and Science. Hrsg. von Lorraine Daston. New York: Zone Books, 2008. S. 9-24, hier S. 15: „The language of things derives from certain properties of the things themselves, which suit the cultural purpose for which they are enlisted“. Vgl. zu den Einwänden gegen diese Sichtweise Adam Bencard und Thomas Söderqvist: Do things talk? In: The Exhibition as Product and Generator of Scholarship. Hrsg. von Susanne Lehmann-Brauns, Christian Sichau und Helmut Tischler. Berlin: Max-Planck-Institut für Wissenschaftsgeschichte, 2010 [Preprint, Bd. 399]. S. 93-102; sowie Thomas Söderqvist: Do things really talk? Are there really hidden stories in objects? Can you write the biography of an artefact? In: Hidden Stories. What do medical objects tell and how can we make them speak? Abstract-Book zur gleichnamigen 16th Biennial EAMHMS Conference vom 13. bis zum 15. September 2012 am Medizinhistorischen Museum der Charité, Berlin. S. 5-12.

5 Einen sinnfälligen Eindruck vom Zustand der sich entwickelnden Disziplin gibt das überzeugend gestaltete Handbuch der Exponatik. Vgl. dazu Fritz Franz Vogel: Das Handbuch der Exponatik: Vom Ausstellen und Zeigen. Köln: Böhlau, 2012. Dass die Unübersichtlichkeit erfreuliche Folgen hervorgerufen hat, illustriert auch die Förderrichtlinie des Bundesministeriums für Bildung und Forschung „Die Sprache der Objekte - Materielle Kultur im Kontext gesellschaftlicher Entwicklungen“ vom 28. März 2012, abgedr. im BAnz AT vom 11. April 2012. Vgl. ebd. die Liste der förderfähigen Themenfelder. 
lungsraum -, in denen sich ein und dasselbe Ding in verschiedenen Zusammenhängen befindet und dementsprechend auch verschiedene Bedeutungen haben kann. Diese Räume lassen sich beschreiben als „Kontexte, die aus Wissen und Dingen gebildet werden". ${ }^{6}$ Und aus diesen Kontexten resultiert, als was das einzelne Ding angesehen wird: als Unikat oder als Stellvertreter, als materielle Kostbarkeit oder als symbolischer Tand. In einem simplen Sinne sind die Dinge, was sie sind. Aber es gibt keinen kontextfreien Raum, in dem ein Betrachter sie antreffen könnte als nur dieses. ${ }^{7}$ Das gilt von Anfang an: Kein Ding existiert kontextlos. Unvermeidlich bezeugt es seinen Ursprung wie seine Funktion und ist in diesem Sinne Überrest einer Situation, Fragment eines Kontextes. Um diesen allerdings in einer Ausstellung sichtbar zu machen, ihn mithilfe des Dings zu vermitteln, bedarf es der „Erläuterung durch Re-Kontextualisierung“. ${ }^{8}$ Das Objekt wird im Raum zwischen andere Dinge und in eine Reihe von Zuschreibungen platziert. Auf diesem Umstand beruhen die Möglichkeiten musealer Präsentation in Ausstellungen.

Die Kunstwerke, von denen Mieke Bal spricht, bilden da keine Ausnahme. Sie liegen als Ausgangspunkt der Betrachtung nahe, nicht nur weil ihr Abstand zu den ,Dingen‘ gemeinhin als beträchtlich gilt, sondern auch weil „das Kunstmuseum als die eigentliche, kanonische Museumsgattung“9 noch immer unsere Vorstellungen dominiert. An Kunstausstellungen lässt sich studieren, welche beiden Strategien den musealen Umgang mit den Dingen bestimmen, nämlich Reduktion und Rhetorik.

\footnotetext{
6 Gottfried Korff: Betörung durch Reflexion: Sechs um Exkurse ergänzte Bemerkungen zur epistemischen Anordnung von Dingen. In: Dingwelten: Das Museum als Erkenntnisort. Hrsg. von Anke te Heesen und Petra Lutz. Köln: Böhlau, 2005. S. 89-107, hier S. 96.

7 Das illustriert die Argumentation von Söderqvist in wünschenswerter Deutlichkeit: Er plädiert dafür, die Dinge nicht zu anthropomorphisieren, sondern den Menschen ding-ähnlicher zu verstehen; „rather than making the world more like us [...] see ourselves more like the world“. Die Bedeutung dieser Forderung nach einem metaphorisch weniger belasteten Sprechen beschreibt er wie folgt: „It will mean abandoning the comfortable realm of social and cultural studies, and instead working to connect scientific and cultural explanations“. Söderqvist verlässt den einen Raum (,realm') und begibt sich in einen anderen. Nicht die Tätigkeit verändert sich, sondern deren Bedeutung. Vgl. dazu Söderqvist: Do things really talk?, S. 11 .

8 Gottfried Korff: Zur Eigenart der Museumsdinge [1992]. In: Museumsdinge: deponieren - exponieren. Hrsg. von Martina Eberspächer, Gudrun Marlene König und Bernhard Tschofen. Köln: Böhlau, 2002. S. 140-145, hier S. 143.
}

9 Anke te Heesen: Theorien des Museums. Hamburg: Junius, 2012. S.15. 
Man muss kein Museumspädagoge sein, um zu wissen, dass eine gute Führung das meiste von dem, was ausgestellt wird, schlicht übergeht. Der Führer zeigt an ausgewählten Bildern, was es im einzelnen Bild überhaupt zu sehen gibt. Wenn er das gut macht, erinnert man sich später an die meisten Bilder, die erklärt worden sind. Und man erinnert sich auch, hinsichtlich welcher Eigenschaften die Beispiele, die man gesehen hat, die Bilder vertreten, die man nicht gesehen hat. Der Führer wählt nicht nur einzelne Bilder aus, sondern auf diesen wiederum einzelne Details, deren Bedeutung er expliziert.

Die Reduktion wird durch Rhetorik ergänzt: Das Vorgehen pars pro toto gehört in der Rhetorik wie in der Didaktik zu den wesentlichen Verfahren der Darstellung. Das ist nicht weiter verwunderlich, denn beide führen ihre methodologischen Konstrukte auf Annahmen über Aufnahmefähigkeit und Aufmerksamkeit zurück.

Und weil es eben darum in Museen und Ausstellungen geht - um die Lenkung von Aufmerksamkeit im Rahmen begrenzter Aufnahmefähigkeit -, verhält es sich mit den Dingen in Ausstellungen nicht anders als mit Kunstwerken. Zwar sind die Dinge, mit denen man im Alltag umgeht (oder mit denen sich Wissenschaftler im Rahmen ihrer Forschungen beschäftigen), in der Regel nicht dazu bestimmt, öffentlich angeschaut zu werden. Aber auf dem Weg, auf dem ein solches Ding in eine Ausstellung gelangt, um dort gezeigt $\mathrm{zu}$ werden, tritt dieser Aspekt in den Hintergrund. Er verliert sich deshalb nicht. Man kann sagen: Die Dinge werden den Bildern ähnlich. ${ }^{10}$ Auch die Führer in einer Wissenschaftsausstellung zeigen beileibe nicht alles, was es zu sehen gibt.

Zwischen der Reduktion der Führer und der Rhetorik der Ausstellungsmacher besteht kein Widerspruch. Die Ausstellungsmacher behaupten (zumindest implizit), die Gesamtheit der Dinge sei sinnvoll, weil gerade die von ihnen getroffene Auswahl geeignet sei, eine bestimmte Sache sichtbar zu machen, darzustellen. Die Gesamtheit der Dinge ergibt ein geschlossenes Ganzes, die Ausstellung.

Explizit gemacht wird dies gewöhnlich mit gesteigerter Rhetorik. In einer Wissenschaftsausstellung zum Beispiel mit der Behauptung, die Ausstellung erzähle „die spannenden Geschichten, die sich hinter den ,Dingen des Wissens“ verbergen“. ${ }^{11}$ Der so angesprochene Zusammenhang ist der zwischen der Auswahl der Dinge - ein Ergebnis von Reduktion - und ihrer (gemeinsamen) Bedeutung - das

10 Eine lohnende Frage lautet, wie weit diese Ähnlichkeit reicht. Als Ausgangspunkt für solche Überlegungen eignet sich Korff: Eigenart der Museumsdinge; sowie Peter Geimer: Über Reste. In: Dingwelten: Das Museum als Erkenntnisort. Hrsg. von Anke te Heesen und Petra Lutz. Köln: Böhlau, 2005. S. 109-118.

11 Georg-August-Universität Göttingen (Hrsg.): Dinge des Wissens: Flyer zur Ausstellung zum 275. Jubiläum der Georg-August-Universität Göttingen. Göttingen: ohne Verlag, 2012. 
Ergebnis der Inszenierung im Raum. Die Dinge sind in der gegebenen Konstellation notwendig, um die Geschichte zu erzählen.

Um diese Art von Rhetorik hat sich eine Diskussion entwickelt, die die Implikationen der Metaphorik betrifft. Denn die Zuschreibung der Eigenschaften an die Dinge - belehrend zu sein oder Geschichten zu erzählen - ist im wörtlichen Sinne problematisch. Das heißt auch: Die Kunst der Ausstellung besteht zu einem nicht unerheblichen Teil darin, die Auswahl der Dinge so zu treffen und ihre Anordnung so vorzunehmen, dass sich das rhetorische Problem lösen lässt. Und das schließt ein, keine Grenze festzulegen, über die hinaus eine Reduktion nicht sinnvoll ist. Es geht vielmehr darum, das Verhältnis zwischen Reduktion und Rhetorik so zu gestalten, dass die Aussage möglichst überzeugend und der Anblick der Dinge möglichst sinnfällig wirkt.

Damit ist offensichtlich, dass Ausstellungsmacher und Führer dasselbe Problem auf dieselbe Weise lösen. Was sich verändert, ist der Kontext. Die Ausstellungsmacher wählen aus einem größeren Vorrat ihre Exponate; die Führer stellen aus den Exponaten wiederum eine kleinere Ausstellung zusammen.

Diese Beobachtung lässt sich leicht weiter verallgemeinern. Die Mehrzahl der Besucher bucht weder Führungen noch benutzt sie elektronische Guides. Es gehört gerade zu den Eigenheiten des Museumsbesuches, einfach durch einen Parcours von Objekten zu schlendern und nur die Exponate näher zu betrachten, die interessant erscheinen. Ganz gleich, welche das sind: Danach darf man behaupten, man hätte die Ausstellung gesehen. Die Mehrzahl der Besucher verhält sich ebenso wie der Führer, nur dass an die Stelle der Kontexte, die er anbietet, das eigene Vorwissen und das eigene Interesse treten. Beide Male wird das Gesehene in einen Kontext gestellt, der nicht offensichtlich ist. Die eigenwilligen Besucher verfügen über dieselbe Medienkompetenz wie der Führer. Sie wählen, und sie stellen Beziehungen zwischen den Exponaten und ihren Kontexten her. Aus diesem Umstand lässt sich folgern, dass alle Besucher fortsetzen, was die Ausstellungsmacher begonnen haben: zum einen Dinge auszusuchen und zum anderen sie mit Bedeutung zu versehen.

Das Zustandekommen der Bedeutung für ein einzelnes Ding beruht dabei jeweils auf dem Verhältnis dessen, was wahrgenommen wird, zu dem, was nicht zu sehen ist oder nicht gesehen wird. Der Bedeutungsübertragung vom Gesehenen auf das Nicht-Gesehene liegt die Annahme zugrunde, dass jedes Exponat nicht nur ein Ding ist, sondern zugleich als ein Beispiel für Beispielhaftigkeit fungiert. Theoretisch verfügt jedes einzelne Ding über das Potential, alle anderen $\mathrm{zu}$ vertreten (was nicht heißt, dass dazu praktisch alle gleich geeignet wären). Es besteht ein Verweisungszusammenhang zwischen Teil und Ganzem. Um ihn einzulösen, greifen beide Seiten, Ausstellungsmacher und Ausstellungsbesucher, auf dieselbe Methodik zurück. Sie betrachten das einzelne Ding immer auch als 
bedeutungsvollen Stellvertreter. Diese Methodik beruht auf dem Zusammenhang zwischen Reduktion und Rhetorik. ${ }^{12}$

In seiner aktuellen akademischen Fassung hat dieser Zusammenhang die Form einer Frage. Es gilt, mit den Worten von Jochen Hennig, als ausgemacht, dass die „Frage der Vereinzelung und Gegenüberstellung von Objekten sowie die Form ihrer Ent- und Neukontextualisierung eine signifikante Kategorie bei der Präsentation universitärer Sammlungen darstellt" “. ${ }^{13}$ Was Hennig für den besonderen Fall universitärer Sammlungen erörtert, lässt die Problemstellung in aller Deutlichkeit hervortreten: Während der Ausstellung wird das Objekt nur unter sehr speziellen Voraussetzungen als Bestandteil einer Sammlung wahrgenommen. Es wird separiert, anderen Objekten hinzugefügt und als Bestandteil dieses neuen Zusammenhangs sichtbar gemacht. Hennigs Frage soll im Folgenden mithilfe des Begriffspaars Reduktion und Rhetorik beantwortet werden. Die beiden Termini ermöglichen, das in Rede stehende Phänomen genauer zu fassen - und ein analytisches Konzept bereitzustellen, um der Frage nachzugehen.

Sammlungen gelten als

Zusammenstellung natürlicher oder künstlicher Gegenstände, die zeitweise oder endgültig aus dem Kreislauf ökonomischer Aktivitäten herausgehalten werden, und zwar an einem abgeschlossenen, eigens zu diesem Zweck eingerichteten Ort, an dem die Gegenstände ausgestellt werden und angesehen werden können. ${ }^{14}$

12 Ich gebrauche die beiden Begriffe vorerst so heuristisch, wie ich sie eingeführt habe. Das entspricht dem Stand der Diskussion. Zu diskutieren wäre etwa der Zusammenhang der unterschiedlichen Perspektiven auf eine Ausstellung: auf der einen Seite die Ausstellungsmacher, die die konzeptionelle Gesamtheit der Präsentation im Blick haben, auf der anderen Seite die Besucher, deren Blick nicht notwendig der Suche nach diesem Konzept gilt, die aber sehr wohl eine konzeptionelle Geschlossenheit wahrnehmen. Gerade hinsichtlich dieser Diskrepanz sollte die Rekonstruktion der Methodik unerlässlich sein, um Aussagen über die Bedeutung aus dieser oder jener Perspektive miteinander vergleichen zu können. Bei Fallstudien dieser Art sollte sich zeigen, ob die hier vorgeschlagene Terminologie überhaupt geeignet ist, die Phänomene zu erfassen, an deren Beschreibung mir gelegen ist.

13 Jochen Hennig: Wissensdinge - Wissensanordnungen - Wissensorte: Zum Ausstellen von Universitätssammlungen. In: Dinge des Wissens: Die Sammlungen, Museen und Gärten der Universität Göttingen. Hrsg. von der Georg-August-Universität Göttingen. Göttingen: Wallstein, 2012. S. 20-29, hier S. 22.

14 Krzysztof Pomian: Der Ursprung des Museums: Vom Sammeln. Berlin: Wagenbach, 1988. S. 16. Vgl. auch ders.: Sammlungen - eine historische Typologie. In: Macrocosmos in Microcosmos. Die Welt in der Stube. Zur Geschichte des Sammelns 1450 bis 1800. Hrsg. von Andreas Grote. Opladen: Leske + Budrich, 1994. S. 107-126. 
Betont wird mit dieser Definition der letzte Zweck der Gegenstände. Es ist das öffentliche Betrachtet-Werden. ${ }^{15}$

Das Ausstellen erscheint als die Fortsetzung des Sammelns, dieses auf jenes ausgerichtet. Und beides beruht auf Auswahl und Variation des Kontextes. Erst wird das Ding gewählt, um in der Sammlung bewahrt, dann ausgesucht, um in der Ausstellung gezeigt zu werden. Der Wahl liegen jeweils andere Kriterien zugrunde. Das Verfahren der Präsentation des Beispielhaften ist (heutzutage) allerdings konstant: Beide Male vertritt das Ding irgendetwas, ohne sich selbst dabei zu verändern. Was es wann bedeutet, wird mitbestimmt durch den Kontext der anderen Dinge, die der Sammlung oder die der Ausstellung. Das Herstellen von Kontext ist ein wesentliches Mittel der Bedeutungsgenerierung. Eine Ausstellung ist der Ort, an dem die Begründungen für die mehrfache Reduktion offengelegt werden. Es werden Dinge gezeigt, und es wird gezeigt, wofür diese Dinge stehen. Sie repräsentieren etwas, indem sie andere Dinge oder eine Idee, eine Geschichte, ein Ereignis, eine Person vertreten. Im Extremfall kann es dazu kommen, dass ein Ding kaum etwas von seiner ursprünglichen Bedeutung behält und eine nahezu neue Bedeutung zugeschrieben bekommt; also Reduktion der einstigen Bedeutung und rhetorische Zuschreibung der neuen. In der Ankündigung der Ausstellung „Dinge des Wissens“ zum 275. Jubiläum der Georg-August-Universität Göttingen findet sich der Vorgang als generelles Problem aller Exponate ausgesprochen: „Die Ausstellung führt eine Vielzahl eindrucksvoller Objekte aus den räumlich und institutionell getrennten Sammlungen zusammen und lädt zu einer neuen Sicht auf die Dinge ein. Was macht sie zu ,Dingen des Wissens"?"16

Im speziellen Göttinger Fall stecken die beiden zentralen Fragen aller (Wissenschafts-)Ausstellungen: Wie stellt man ein Ding so aus, dass man ihm das Wissen ansehen kann, das mit ihm verbunden ist? Wie sieht man ein Ding so an, dass man das Wissen, das mit ihm ausgestellt wird, erkennt? ${ }^{17}$

Anhand von zwei Wissenschaftsausstellungen soll illustriert werden, welche Implikationen diese Fragen verbergen und welche Möglichkeiten der Beantwortung das Konzept ,Reduktion und Rhetorik‘ bietet. Die ausgewählten Dinge sind nichts anderes als Beispiele für Beispielhaftigkeit.

15 An diesem Punkt könnte eine Ausdifferenzierung des Ausstellungsbegriffs ansetzen, die Hennigs Überlegungen aufgreift und fortführt: Zu welchem Zweck werden universitäre Sammlungen angelegt und für welche Öffentlichkeit sind sie bestimmt?

16 Universität Göttingen (Hrsg.): Dinge des Wissens: Flyer.

17 Es handelt sich am Ende um zwei Varianten derselben Frage. Beim jetzigen Stand der methodologischen Überlegungen dürfte es nützlich sein anzuzeigen, dass die Sache sich von mehreren Seiten betrachten lässt. 


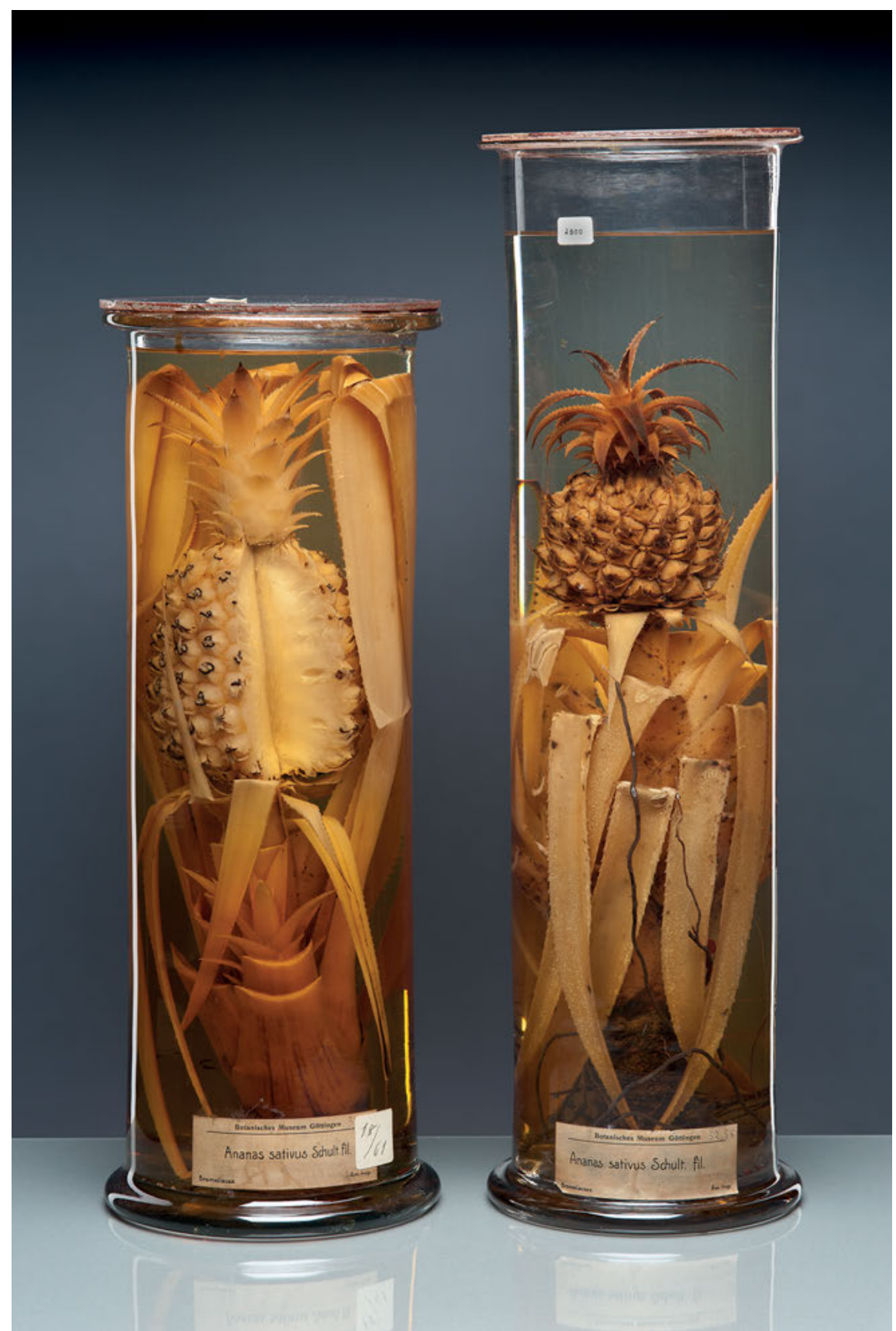

Abb. 3: Alkoholpräparat Ananas, Standglas, um 1900, ca. $50 \mathrm{~cm}$ hoch, Alter Botanischer Garten, Göttingen (Foto: Stephan Eckardt). 
Das erste Beispiel ist eine Ananas (zwei Exemplare davon). Auf den ersten Blick ist klar, um welche Spezies es sich handelt - was gibt oder gäbe es da zu sehen?

Dieser Frage wird im Folgenden kursorisch und trotzdem recht aufwendig nachgegangen. Dabei wird zunächst das Objekt genauer betrachtet. Sodann werden die naheliegenden Kontexte gemustert, um einen Überblick über das Wissen zu geben, das mit dem Ding verbunden ist. Anschließend wird der Gebrauch von Ananas in zwei Ausstellungen analysiert, um vorzuführen, dass der Kontext die Bedeutung eines Exponats nahezu vollständig bestimmt. Diese Überlegungen können auch als Blick in die Werkstatt gelesen werden: Was ist zu bedenken bei der Vorbereitung einer Wissenschaftsausstellung, in der auch ein Glas ausgestellt werden soll, in dem sich eine Ananas befindet?

Bei dem abgebildeten Objekt handelt es sich nicht um zwei Ananas, sondern um zwei Ananas im Glas. Das ist ein erheblicher Unterschied: Frucht, Flüssigkeit, Glas und Beschriftung bilden das auszustellende Objekt. Es stammt aus den Beständen einer botanischen Sammlung. Diese Sammlung dürfte der erste naheliegende Kontext sein. In ihr haben die Gläser ihren Stammplatz. Ein Verweis auf diesen Kontext ist kaum zu unterdrücken. In der Sammlung haben die Gläser nicht den Status von Exponaten, sondern sind Präparate.

Aber was wird in der botanischen Sammlung durch das Präparat vertreten? Es verweist auch hier auf einen anderen Kontext, nämlich den ursprünglichen, und das heißt dieses Mal: auf den natürlichen. Das wird durch die Beschriftung auf dem Etikett angezeigt. Dort ist festgehalten, was zu sehen ist. Der Laie kann erkennen, dass dieser natürliche Kontext für die Botanik von Interesse ist. Das sieht er nicht an der Frucht, sondern am Glas und seiner Beschriftung. Welche der natürlichen Eigenschaften der Frucht die Neugier der Wissenschaft geweckt hat, welche Phänomene also durch die Anwesenheit der Exemplare in der botanischen Sammlung vertreten werden, kann der Laie nicht sagen. Spätestens an dieser Stelle erwartet er eine Information über den Kontext.

Damit sind die für das Sammeln und Ausstellen zentralen Fragen berührt: Warum und wie kommt die Ananas ins Glas? Und warum kommt das Glas, später, in die Ausstellung? Was fand und was findet die Wissenschaft so interessant an der Ananas?

Nun, das ist eine lange Geschichte. Die Kuratoren bereiten in solchen Fällen schon einmal einen Teil der Führung vor - sodass der Besucher dem Wechselspiel von Reduktion und Rhetorik so ausgesetzt werden kann, dass ihm am Ende einleuchtend erscheint, was sie ausstellen wollen. Hier sollen nur die Möglichkeiten einer solchen Führung angedeutet werden, die der Reduktion ebenso wie die der Rhetorik, weshalb die lange Geschichte nicht erzählt wird, sondern mögliche Kontexte und Ausstellungen vorgeführt werden. 
Christoph Kolumbus hat im November 1493 aus Guadeloupe eine Frucht mitgebracht, für die man zu Hause noch keinen Namen hat. Der Geschichtsschreiber der spanischen Krone gibt davon Zeugnis und vermerkt, dass der König der Frucht höchstes Lob spendete. ${ }^{18}$ Der Name ,Ananas‘ wird einem Franzosen zugeschrieben, André Thévenet, der ihn 1555 in einem Reisebericht aus dem Guaraní-Wort „nana‘ ableitete, was so viel wie „köstliche Frucht” bedeutet. ${ }^{19}$ Während zu dieser Zeit die Ananas in Südamerika schon als Nahrungs- und Heilmittel verbreitet war, ${ }^{20}$ gehörte der Genuss der Frucht in Europa noch einige Zeit zu den königlichen Privilegien. Das ist an ihrem englischen Namen zu erkennen: King Pine.

Eine ganze Reihe von Königen machte mit der Frucht Politik. Charles II. vergab an seiner Tafel einzelne Stücke der Ananas zum Verkosten und zeichnete so die Bedachten aus. ${ }^{21}$ George I. nutzte die Ananas, um mit dem Technologievorsprung vor ausländischen Gästen anzugeben: Es sei gelungen, die exotische Frucht im Lande zu ziehen. ${ }^{22}$ Um die erste in England gewachsene Ananas gibt es einen Streit, der sich nur durch die Verbindung von kunsthistorischem mit botanischem Sachverstand lösen lässt.

Hendrick Danckerts malte vermutlich 1662 den Regenten bei der Übernahme einer Frucht vom königlichen Gärtner. Theodor Netscher portraitierte 1720 eine Ananas, die im Garten von Sir Matthew Decker gewachsen war. ${ }^{23}$ Danckerts' Bildtitel dürfte reine Propaganda sein. Die Frucht, die er malte, stammte aller Wahr-

18 Vgl. Peter Martyr von Anghiera: Acht Dekaden über die neue Welt. Bd. 1. Übers. von H. Klingelhöfer. Darmstadt: Wissenschaftliche Buchgesellschaft, 1972. S. 201.

19 Vgl. Helmut Genaust: Etymologisches Wörterbuch der botanischen Pflanzennamen. 3. Aufl. Basel: Birkhäuser, 1996. S. 60.

20 Vgl. Gunther Franke (Hrsg.): Nutzpflanzen der Tropen und Subtropen. Bd. 2. Stuttgart: Ulmer, 1994. S. 172.

21 Vgl. John Evelyn: The Diary. Hrsg. von E. S. de Beer. London: Oxford UP, 1959. S. 523. Hier der Eintrag zum 14. August 1668: „There was of that rare fruit called the King-Pine, (growing in Barbados \& W. Indies), the first of them I had ever seen; His Majesty having cut it up, was pleasd to give me a piece off his owne plate to tast off“. Herv. im Orig.

22 Vgl. Lady Mary Wortley Montagu: The Complete Letters. Bd.1. Hrsg. von Robert Halsband. Oxford: Clarendon Press, 1965. S. 290. Hier der Brief vom 17. Dezember 1716: „I had more reason to wonder that night at the King's Table. There was brought to him from a Gentleman of this Country 2 large Baskets full of ripe Oranges and Lemons of different sorts, many of which were quite new to me, and what I thought worth all the rest, 2 ripe Ananas's, which to my taste are a fruit perfectly delicious. You know they are naturally the growth of the Brazil, and I could not imagine how they came here but by enchantment. Upon Enquiry I learnt that they have brought their Stoves to such perfection, they lengthen the Summer as long as they please, giving to every plant the degree of heat it would receive from the Sun in its native Soil.“

23 Vgl. dazu Julius L. Collins: The Pineapple: Botany, Cultivation, and Utilization. London: Hill, 1960. S. 22-24. 

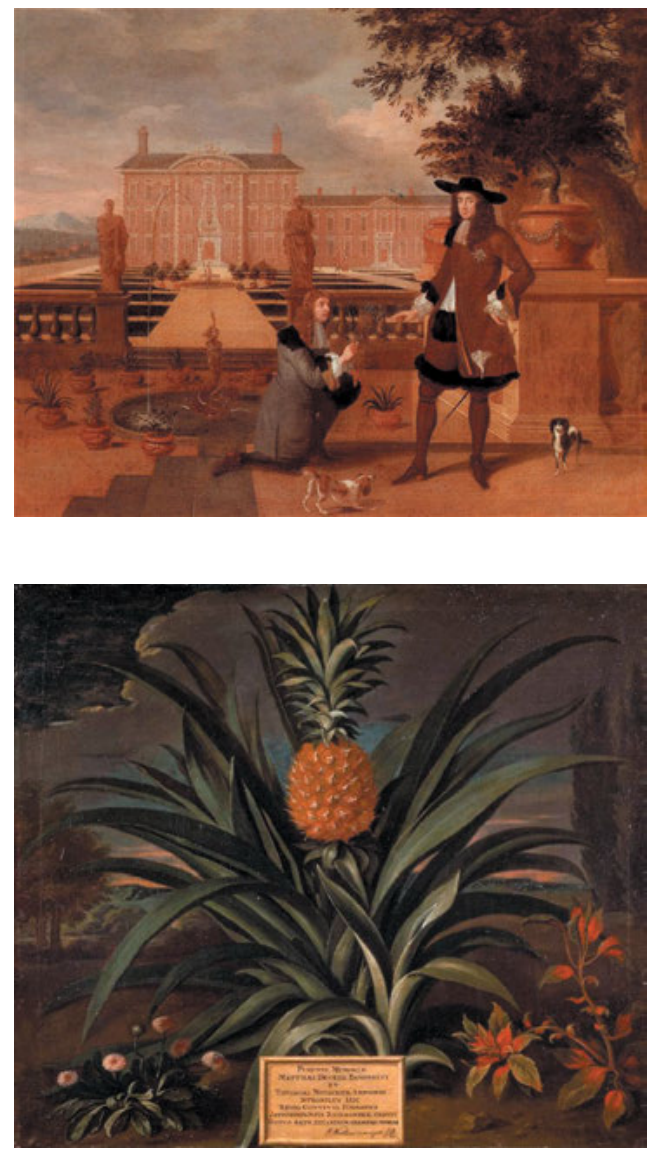

Abb. 4: Hendrick Danckerts: Royal Gardener John Rose presenting a pineapple to King Charles II. (1675).

Abb. 5: Theodor Netscher: Pineapple grown in Sir Matthew Decker's garden at Richmond, Surrey (1720).

scheinlichkeit nach aus Barbados. Decker dagegen hatte Kontakte zu dem flämischen Kaufmann Le Cour. Er galt den Zeitgenossen als der erste, „dem es gelungen Ananas zu treiben“. ${ }^{24}$ Dazu nutzte er in seinem Leydener Garten eigens eingerichtete Gewächshäuser. Auf dem Kontinent war seinerzeit strittig, ob die Pflanze in Leipzig eher als in Paris zu sehen war. Johann Christoph Volkamer berichtete bereits $1714 \mathrm{im}$ zweiten Teil seines Nürnbergischen Hesperidum, dass es seinem Bruder gelungen sei, das seltene Gewächs zu ziehen. Auch er benutzte die soge-

24 Thomas Andrew Knight: Das Ganze der Ananaszucht, oder die verschiedenen Arten, wie man Ananas gezogen hat und noch zieht, von der ersten Einführung dieser Frucht in Europa bis zu den neuesten Kulturverbesserungen. Weimar: Voigt, 1881. S. 3. Vgl. auch S. 8-12. 
nannten Fensterhäuser. ${ }^{25}$ Seit 1743 ist durch Albrecht von Hallers Pflanzenkatalog aktenkundig, dass es auch im Botanischen Garten Göttingen gelungen war, Ananas anzupflanzen. ${ }^{26}$ Nach 1800 sind den ,verschiedenen Arten, wie man Ananas gezogen hat und noch zieht“, mehrere Fachbücher gewidmet. Die maßgebliche Autorität war Thomas Andrew Knight. ${ }^{27}$ Die ersten Experimente mit Ananas in Dosen wurden nach 1880 auf Hawaii gemacht, ${ }^{28}$ der internationale Handel mit Frischananas kam erst nach dem Zweiten Weltkrieg zustande.

An dieser Skizze einer Kulturgeschichte der Ananas ist mühelos zu erkennen, dass sie nicht nur vom Namen her für das Unbekannte steht, sondern auch von Kolonialismus, Eurozentrismus, vom nationalen Wettbewerb, Manipulationen und Fälschungen, Öffentlichkeitsarbeit und symbolischem Kapital handelt. Das mutet alles sehr aktuell an, eine Ausstellung über die Kulturgeschichte der Ananas liegt in der Luft. Das heutige Wissen über die Pflanze dürfte dort ebenfalls seinen Platz bekommen. Es variiert entsprechend der disziplinären Kontexte und gibt ein deutliches Bild vom Zustand unserer Kultur des Wissens. Auch hier genügen wenige Angaben zum Beleg: Nur wenige Vertreter der Gattung Ananasgewächse wurzeln im Boden; die Exemplare, die sich in den Gläsern befinden, gehören dazu. Aus botanischer Sicht sind das Vorhandensein von Wurzeln und der an einen Zapfen erinnernde Fruchtverband ein wichtiges Gattungsmerkmal. ${ }^{29}$ Der Blütenstand einer Ananas interessiert Botaniker und Mathematiker gleichermaßen. Die einen sprechen von einer Scheinfrucht, die anderen von den Fibonacci-Zahlen. Letzteres ist eine Zahlenreihe, in der sich die Glieder jeweils aus der Summe ihrer beiden Vorgänger ergeben. Mithilfe dieser Reihe lassen sich auch natürliche

25 Vgl. Johann Christoph Volkamer: Continuation der Nürnbergischen Hesperidum. Oder: Fernere gründliche Beschreibung der Edlen Zitronat-Zitronen und Pomeranzen-Früchte [...]. Nürnberg: Selbstverl., 1714. S. 210: „Dieses aber ist gewiß / daß es in meines geliebten Bruders Herrn D. Volkamers schönem Haus-Garten / worinnen / in einem nicht gar zu weitläufftigen Raum / was die vier Welt-Theile von seltenen Gewächsen uns bishero eingereichet / gleichsam eingeschrenket und enthalten / sogleich nur von diesem Gewächs einige Kundschafft erschollen / auch glücklich cultiviret / und zur zeitigsten Frucht gebracht worden / so daß nun durch seinen Fleiß so viel Sorten von dieser Frucht bisher bekannt / auch in schönster Vollkommenheit bey ihm zu finden.“ Man habe „durch Kunst dasjenige ersetzt [...] / was die Hitze der Sonnen-Strahlen / denen etwas nördlichern Ländern an Wachsthum entziehet“.

26 Vgl. Michael Schwerdtfeger: Die Ananas im Königreich Hannover und die Sammlung exotischer Pflanzen im botanischen Garten. In: Dinge des Wissens. Die Sammlungen, Museen und Gärten der Universität Göttingen. Hrsg. von der Georg-August-Universität Göttingen. Göttingen: Wallstein, 2012. S. 172-174, hier S. 172.

27 Vgl. Knight: Ananaszucht.

28 Vgl. Collins: Pineapple, S. 227-228.

29 Vgl. Franke (Hrsg.): Nutzpflanzen, Bd.2. S. 172. 
Wachstumsprozesse beschreiben, bei der Ananas etwa das Zustandekommen der Scheinfrucht - geometrisch gesprochen: ihrer Form. ${ }^{30}$

Die Betriebsökonomen, Wirtschaftsgeographen und Spezialisten für tropische Landwirtschaft befassen sich mit der Pflanze ebenso wie Ernährungswissenschaftler und Mediziner. Die Ananas ist für ihren hohen Zuckergehalt und durchschnittlich sieben mg Vitamin C auf $100 \mathrm{~g}$ essbare Bestandteile bekannt. ${ }^{31}$ Ihr Saft enthält Salicylsäure, das ist der hauptsächliche Wirkstoff etwa im Aspirin. Dass die Philosophen seit Langem mit der Ananas vertraut sind, liegt an ihrem besonderen Geschmack. Bekannt ist das Problem spätestens seit John Lockes An Essay Concerning Human Understanding, in dem es heißt:

[B]ut yet I think it will be granted easily, that if a child were kept in a place where he never saw any other but black and white, till he were a man, he would have no more ideas of scarlet or green, than he that from his childhood never tasted an oyster or a pine-apple has of those particular relishes. ${ }^{32}$

Mit anderen Worten: Seit 1689 ist die Ananas ein einschlägiges Beispiel für die These, dass unser abstraktes Wissen in hohem Maße erfahrungsabhängig ist.

Das wird durch den kurzen kultur- und wissenschaftsgeschichtlichen Abriss in aller Deutlichkeit belegt. Nichts von dem Gesagten ist der Ananas anzusehen. Von Natur aus hat ,die Frucht' keine erkenntnisfördernden Eigenschaften. Vielmehr wären all diese für die Bedeutung der Frucht relevanten Kontexte in einer Ausstellung erst einmal darzustellen, um den Zusammenhang zum Wissen und seiner Geschichte sichtbar zu machen. Jeder von ihnen hat das Potential, den Anblick der Ananas zu verändern. Die Folge der Kontexte, in denen die Frucht interessant erschien, bildet die Entwicklung von Wissenschaft und ihren Bedingungen ab. Das gilt in dem allgemeinen Sinne, in dem etwa die Entwicklung einer Disziplin dokumentiert wird, und in dem konkreten, ortsgebundenen Sinn, in dem sich diese Entwicklung an einer Universität ereignet. Aber die wenigsten dieser Kontexte führt das Objekt mit sich, man muss sie sichtbar machen.

Im konkreten Fall sind einige der Kontexte relativ dauerhaft mit der Frucht verbunden, was ihren Status als Ding (des Wissens) entscheidend verändert. Die Göttinger Ananas sind dabei keine Seltenheit, an anderen Orten wird die gleiche Frucht aufbewahrt.

30 Vgl. dazu Stephan Thieß: [o. T.] www.unikik.uni-hannover.de/downloads/mathematik_ diskret.pdf. Skript zum Kurs Diskrete Mathematik der Gauß AG der Leibniz Universität Hannover, Wintersemester 2005/2006. (11. März 2011).

31 Vgl. dazu Wolfgang Franke: Nutzpflanzenkunde: Nutzbare Gewächse der gemäßigten Breiten, Subtropen und Tropen. 4. Aufl. Stuttgart: Thieme, 1989. S. 311-313.

32 John Locke: An Essay Concerning Human Understanding. Hrsg. von Roger Woolhouse. London: Penguin Books, 1997. S.111. 


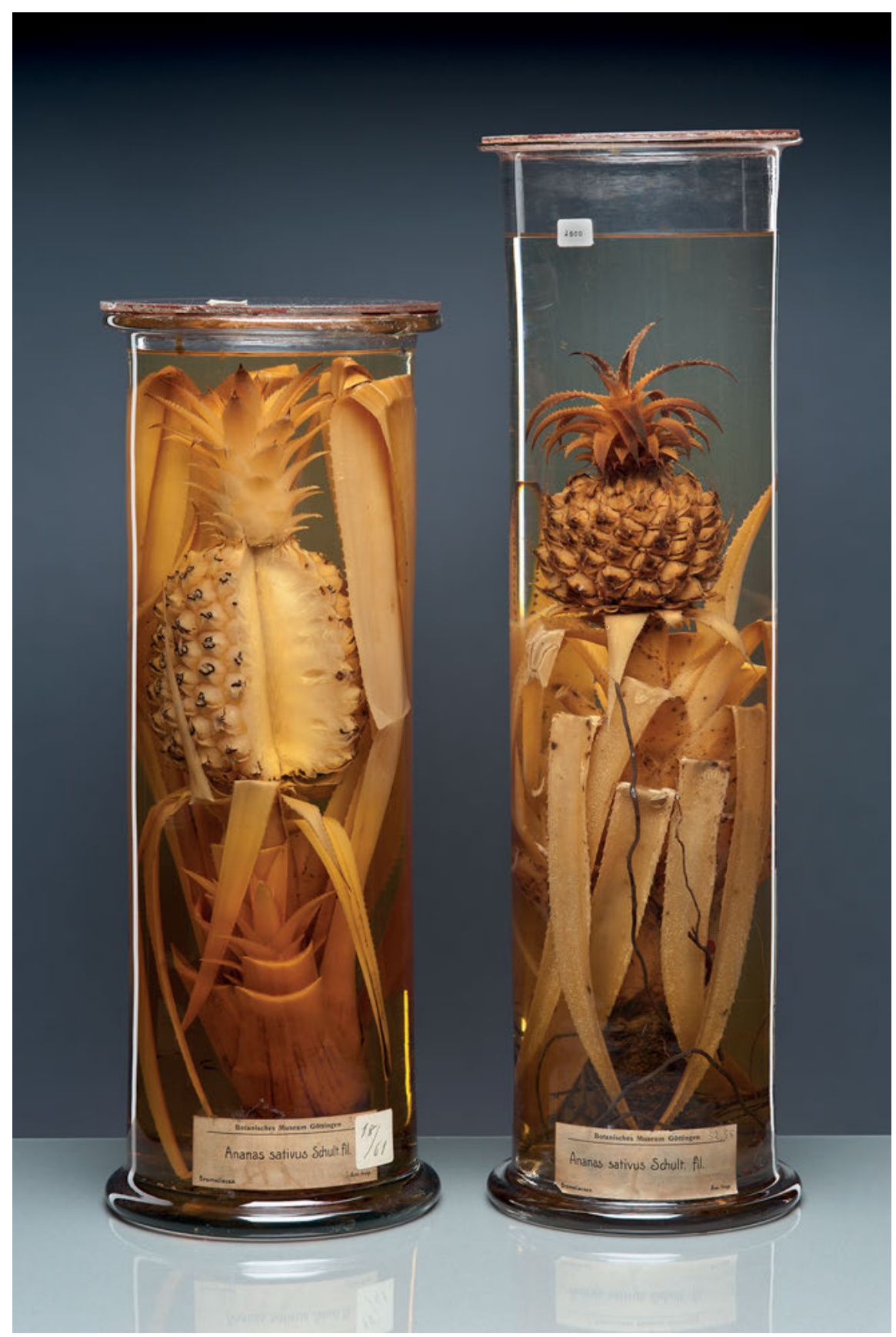

Abb. 6: Alkoholpräparat Ananas, Standglas, um 1900, ca. 50 cm hoch, Alter Botanischer Garten, Göttingen (Foto: Stephan Eckardt). 


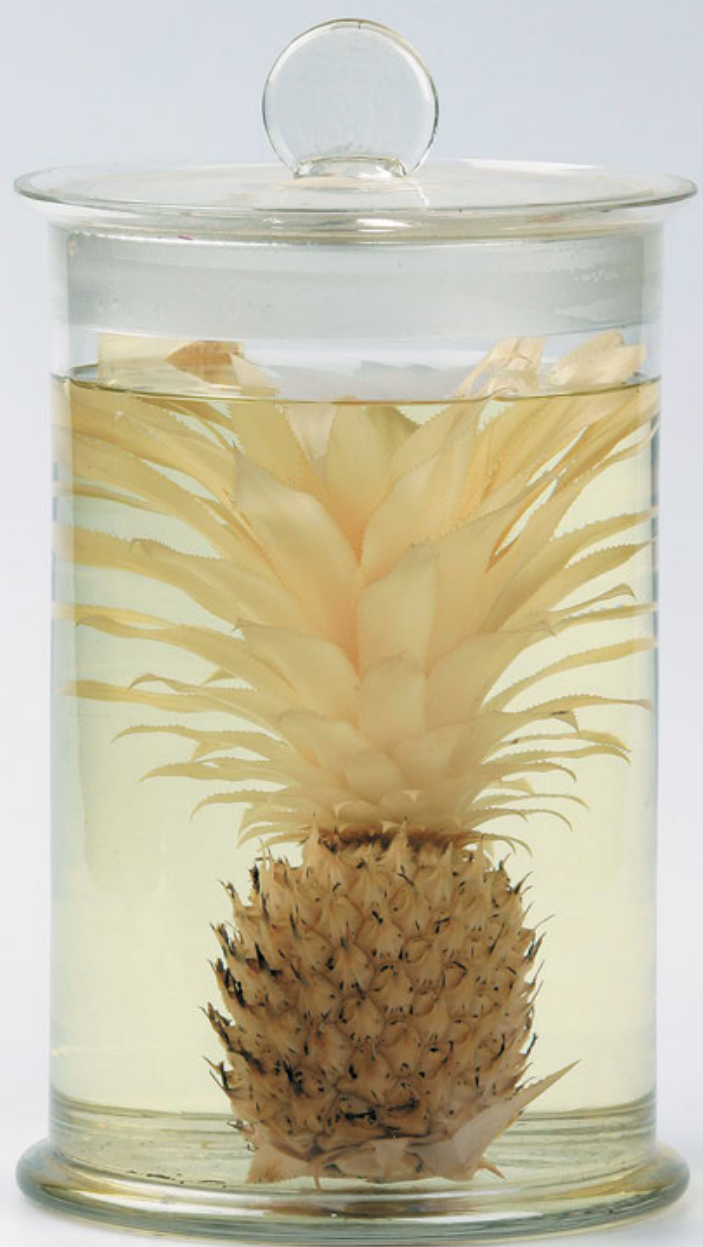

Abb. 7: Feuchtpräparat, Ananas Comosus (L.), Merr., Botanische Sammlung Erlangen (Foto: Georg Pöhlein). 
In allen drei gezeigten Fällen kamen die Früchte um 1900 in ihr Glas. Auch wenn das Aussehen der einzelnen Früchte deutlich voneinander abweicht, gleicht sich die Struktur des Objekts, dessen Kern sie bildet. Selbst die Beschriftung, deren Wortlaut erheblich voneinander abweicht, folgt derselben Taxonomie, die Namen sind Synonyme. Das Objekt Ananas, wie es hier zu sehen ist, signalisiert seinen Zusammenhang zum Wissen unvermeidlich. Das liegt an seiner Beschaffenheit, seinem Aufbau - Glas, Flüssigkeit, Frucht, Beschriftung. Am Glas und seiner Beschriftung ist $\mathrm{zu}$ erkennen, dass Natur konserviert und klassifiziert worden ist, zweifellos in didaktischer Absicht. Objekten dieser Art kann man die Einheit von Forschung und Lehre ansehen: Dass sie überhaupt beschriftet werden konnten, ist ein Resultat der Forschung, die die zur Einordnung notwendigen Kriterien hervorbrachte. Auch die Präparation selbst, von den Werkzeugen über die Gefäße und Chemikalien bis hin zu den nötigen Handgriffen, ist Bestandteil von Fachwissen.

Insofern dokumentiert das Objekt einen historischen Stand der Botanik. Es vertritt eine Zeit, in der das Fach mit „Fragen über die Abgrenzung von Arten, Gattungen wie Familien und die natürliche Verwandtschaft“33 beschäftigt war. Diesen Kontext führt das Objekt mit sich, das Göttinger Exemplar zeigt auch an, in welche Sammlung es gehört. Mit der Frucht wird Wissen aufgehoben, keine Nahrung.

Die Präsentation des Wissens auf dem Etikett folgt Linné, mit dessen System Reduktion und Rhetorik als Prinzip Einzug in die Botanik halten. An die Stelle der grenzenlosen Anhäufung von Pflanzen tritt eine Sammlung der Arten, ein „Tableau universeller, taxonomischer Beziehungen“, ${ }^{34}$ das die Einordnung jeder Pflanze gestatten soll. Ein einzelnes Exemplar vertritt die Art. Auf dem Weg ins Glas ist die Ananas von der Frucht zum Präparat und damit zu einer Darstellung ihrer selbst geworden. Das ist ein (erträgliches) zeichentheoretisches Paradox, das „Objekt ist Repräsentation“..35 Jeder Ausstellungsbesucher weiß, dass eine konkrete Frucht zu sehen ist, an der etwas zu erkennen ist, das nicht nur für sie gilt. Diese Betrachtungsweise steht der des Alltags konträr gegenüber. Beim Ananas-Einkauf blickt man gewöhnlich die einzelne Frucht gerade auf die Eigenschaften hin an, die sie von anderen unterscheidet.

33 Hans Solereder: Systematische Anatomie der Dicoyledonen. Stuttgart: Enke, 1899. S. V.

34 Peter J. Bräunlein: Material Turn. In: Dinge des Wissens: Die Sammlungen, Museen und Gärten der Universität Göttingen. Hrsg. von der Georg-August-Universität Göttingen. Göttingen: Wallstein, 2012. S. 30-44, hier S. 38.

35 Hans-Jörg Rheinberger: Objekt und Repräsentation. In: Mit dem Auge denken. Strategien der Sichtbarmachung in wissenschaftlichen und virtuellen Welten. Hrsg. von Bettina Heintz und Jörg Huber. Zürich: Ed. Voldemeer, 2001. S. 55-61, hier S. 59. Herv. im Orig. 


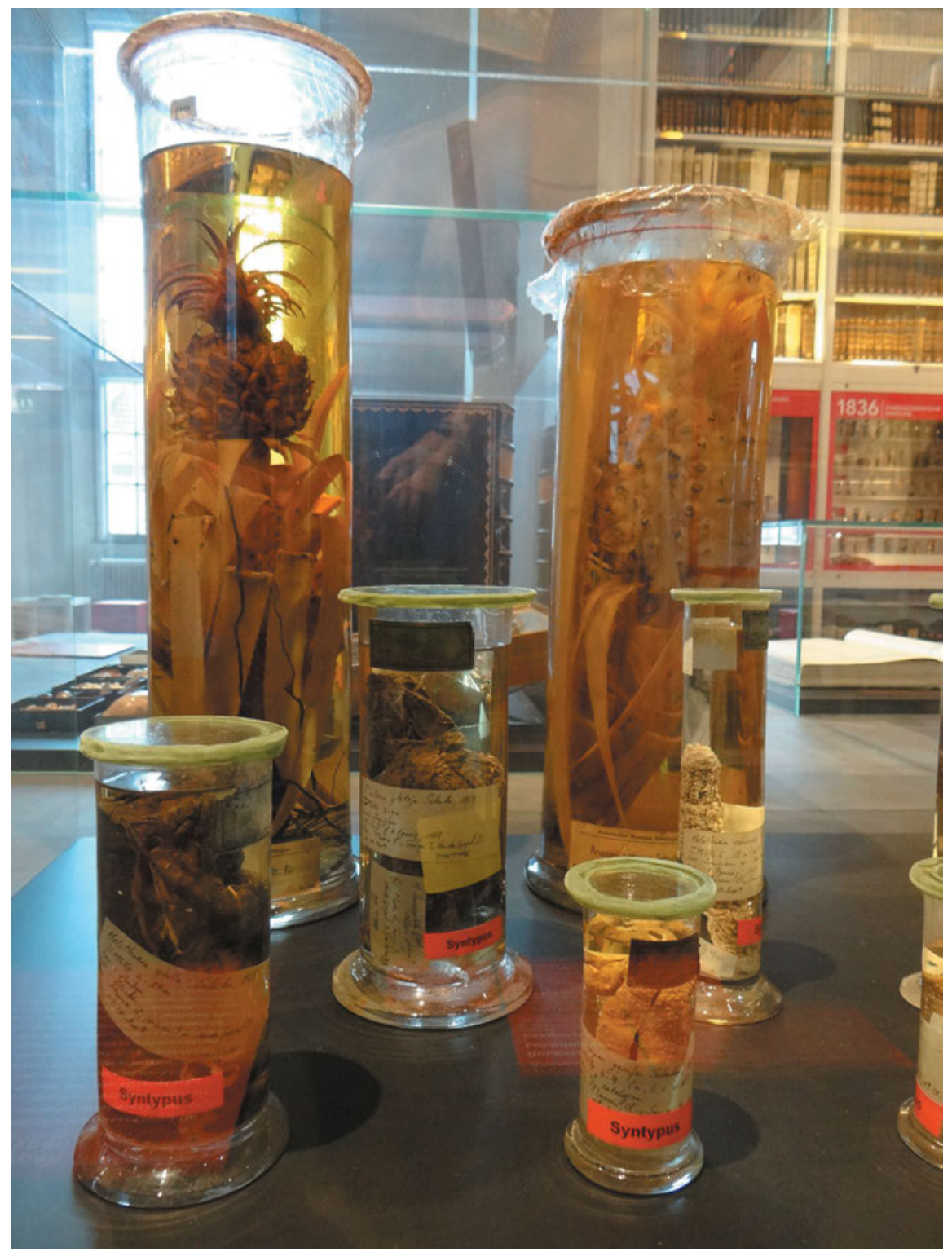

Abb. 8: Ananas in der Göttinger Ausstellung. Enger Kontext: Feuchtpräparate (Foto: Holger Helbig). 
Übernimmt man das Präparat in eine Ausstellung, fungiert es dort als ein Zeichen. Es wird zum Exponat und damit verfügbar für rhetorische Zugriffe. Es sagt weniger über sich selbst als über seinen Kontext aus. Welcher das ist, hängt von der Ausstellung ab.

Ein erster Blick auf die Göttinger Ausstellung zeigt, dass die beiden Präparate zusammen mit anderen Präparaten stehen. Das verweist zum einen auf eine bestimmte konservatorische Arbeitstechnik und ihr Ergebnis: Feuchtpräparate. Zum anderen verweisen verschiedene Präparate auf verschiedene Sammlungen. Im konkreten Fall handelt es sich um Seegurken und Ostracoda, winzige Krebse.

Mit der Präparation wird „der Sammlungsgegenstand einem neuen Kontext zugeführt“" ${ }^{36}$ Er variiert nach Anlage und Ausrichtung der Sammlung; im Fall der Ananas ist es der Kontext der Botanik. Das Interesse am ausgewählten Objekt ist bedingt durch das in der Botanik , angesammelte' Wissen. Es soll an die nachfolgenden Wissenschaftler vermittelt werden.

Einmal zum Bestand der botanischen Sammlung gehörig, trägt das Präparat zu deren Profil bei. Das ist für Göttingen ganz erheblich, denn der Verweis gilt auch der Sammlung von Ananasgewächsen, die mit etwa 3000 Arten, Formen und Herkünften zur Geltung der Göttinger Universität beiträgt. ${ }^{37}$

Das wird aber gerade nicht betont, stattdessen findet sich in der Vitrine noch eine Xylothek (Holzbibliothek). Damit verdeutlicht die Anordnung der Präparate, dass die hier aus ihrem Sammlungskontext gelösten Exponate eine abstrakte Gemeinsamkeit repräsentieren sollen: Sammlungen unterliegen fachspezifischen Ordnungen. Weil das schwer zu sehen ist, wird es auf der entsprechenden Legende expliziert.

Die roten Täfelchen arretieren den Kontext, indem sie ihn explizieren. Ausgestellt werden soll der Zusammenhang zwischen Systematik und Disziplin, die Idee wissenschaftlicher Ordnung. Das einzelne Exponat erfüllt dementsprechend eine Aufgabe, welche die Frucht und das Präparat in ihren ursprünglichen Kontexten nicht erfüllen können, zu der das Objekt nichtsdestotrotz geeignet ist. ${ }^{38}$ Dass jedes Objekt zur Sichtbarmachung der Idee beiträgt, ist an der Vielzahl der

36 Jochen Brüning: Wissenschaft und Sammlung. In: Bild, Schrift, Zahl. Hrsg. von Sybille Krämer und Horst Bredekamp. München: Fink, 2003. S. 87-113, hier S. 89.

37 Vgl. Schwerdtfeger: Ananas, S. 174.

38 In der Göttinger Sammlung wurde auf den roten Täfelchen auch die heutige fachliche Relevanz des Objekts ausgesprochen. Es hieß dort über die Ananas: „Sie waren vor der Einführung der Farbfotografie, des Internets und von Frischimporten wertvolle Instrumente der Wissensvermittlung“. Im Klartext: Sie sind es nicht mehr. - Ich bemühe mich zu zeigen, dass dies ein Irrtum ist. Die Ananas im Glas sind von botanischen zu wissenschaftshistorischen Objekten geworden, nach wie vor handelt es sich um wertvolle Instrumente der Wissensvermittlung. 


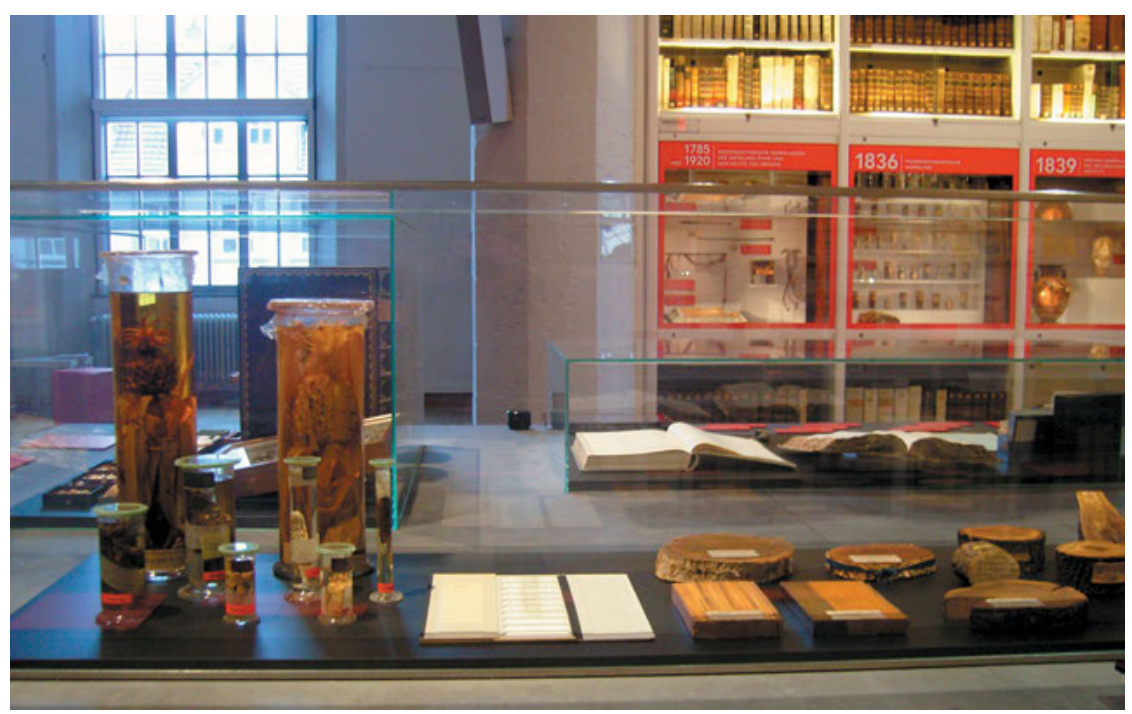

Abb. 9: Ananas in der Göttinger Ausstellung. Weiterer Kontext: Vitrine. Beispiele für typologische Ordnungen (Foto: Holger Helbig).

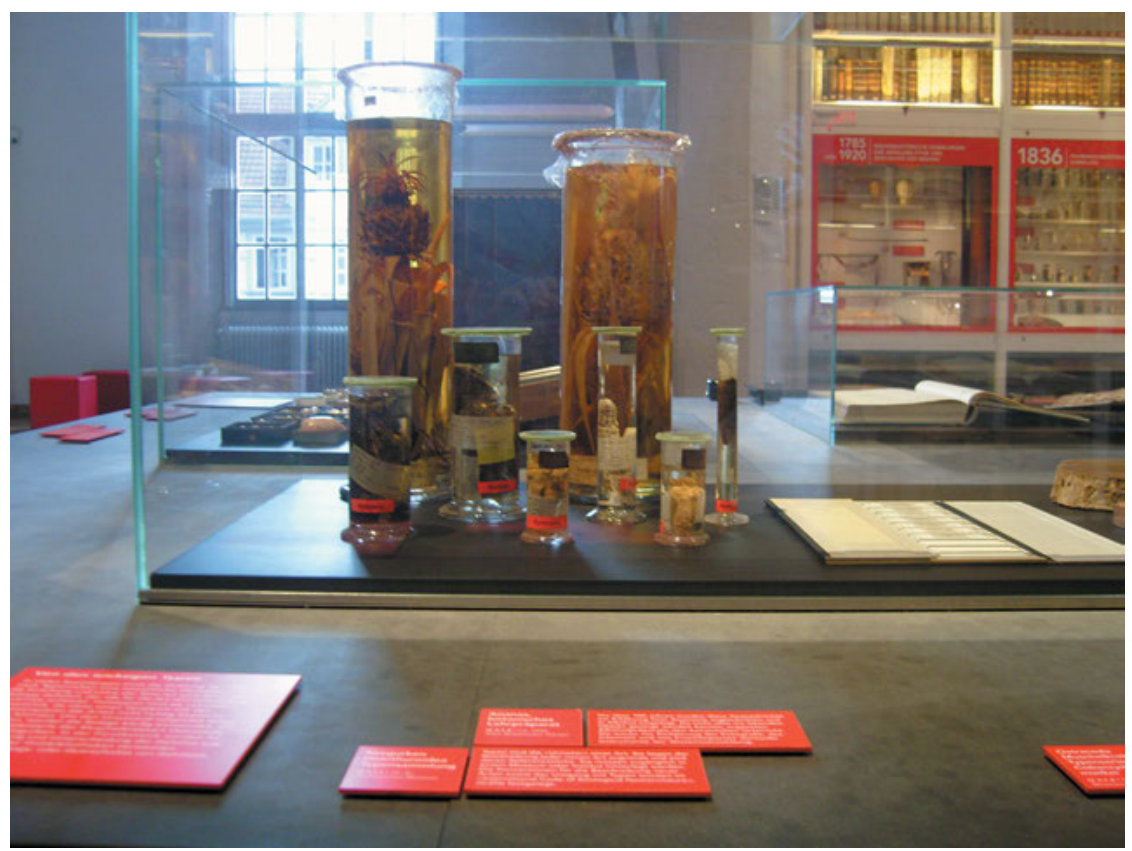

Abb. 10: Ananas in der Göttinger Ausstellung. Explizierte Kontexte: wissenschaftliche Ordnung(en) (Foto: Holger Helbig). 


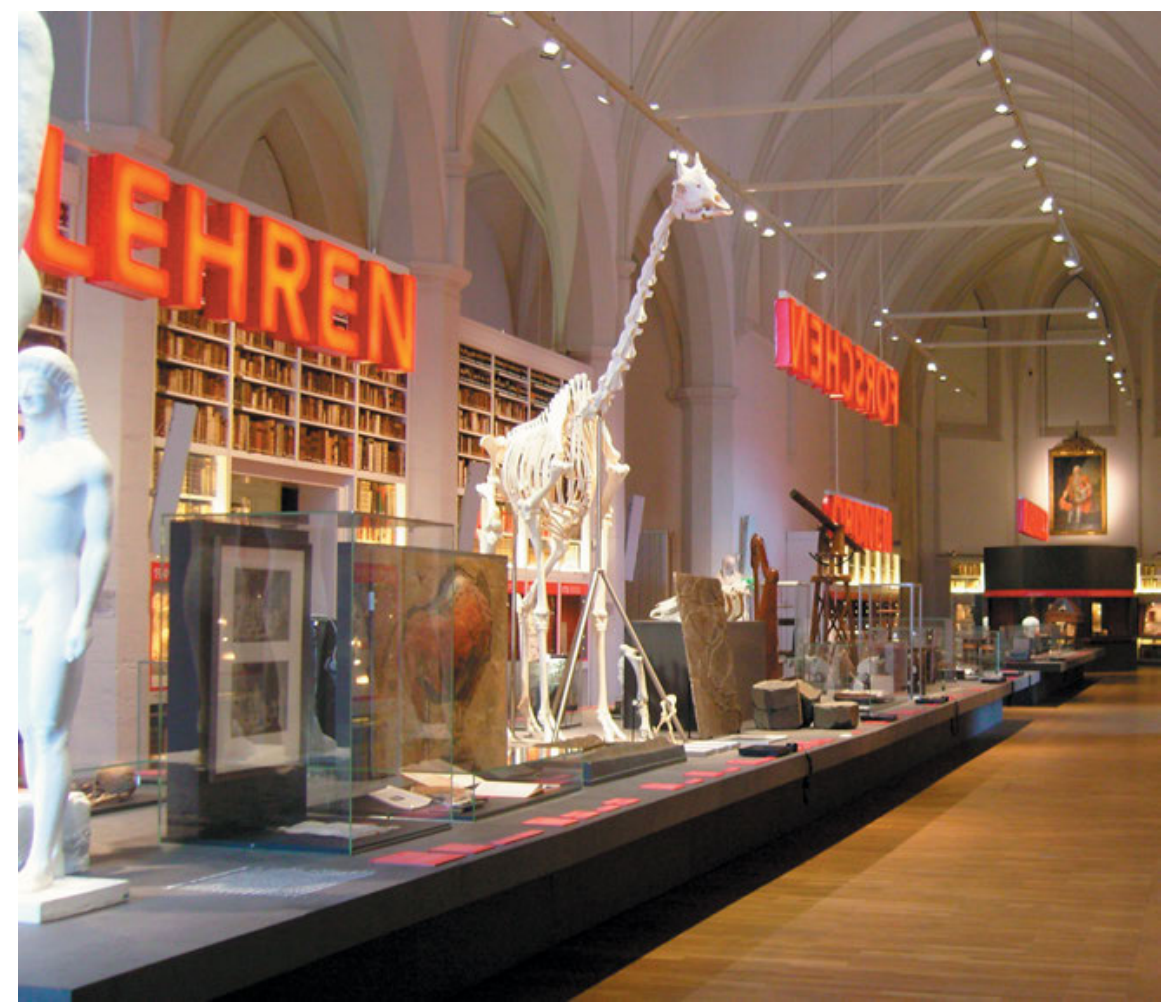

Abb. 11: Ananas in der Göttinger Ausstellung. Weitere Kontexte: Lehren, Forschen (Foto: Holger Helbig).

kleinen roten Täfelchen zu erkennen, die einer größeren, übergeordneten roten Tafel zugeordnet sind. In dieser Konstellation kommt die rhetorische Figur der enumeratio zum Tragen, was auch bedeutet: Die Möglichkeit, mit weniger oder mehr Beispielen auszukommen, ist offensichtlich.

Die Folge der Kontexte lässt sich von der Beschriftung über die Platzierung im Raum, zum Text auf der Tischfläche, der Stellung in der Reihe der Exponate usw., bis zum Titel der Ausstellung verfolgen.

Weitere Kontexte sind etwa die in Leuchtschrift angezeigten Schritte: Sammeln, Ordnen, Forschen, Lehren und nicht zuletzt der Ausstellungsort. Die Göttinger Ausstellung fand in der Paulinerkirche statt, die einen wirkungsvollen Kontext stiftete. Einerseits begrenzte sie durch ihre kulturelle Besetztheit (ein sakraler wie ein säkularisierter Raum) und architektonische Beschaffenheit (nur ein einziger Ausstellungsraum von großer Höhe, der durch die aktuelle bibliothekarische Nutzung bereits gestaltet ist) die Möglichkeiten der räumlichen Präsentation. An- 
dererseits fügte sie die Ausstellung zum Universitätsjubiläum in eine Tradition ein, die die Institution mit der Öffentlichkeit der Stadt und des Landes verbindet. Seit ihrer Gründung ist die Geschichte der Göttinger Universität mit dieser Kirche verbunden, die Ausgestaltung von Jubiläen spielt dabei ebenso eine Rolle wie die Präsentation von Sammlungen. ${ }^{39}$

Es scheint, als füge sich die Nutzung der Ananas im Glas auf naheliegende und einleuchtende Weise in diese Kontexte. Es ist aber bei Weitem nicht die einzige Möglichkeit, von einem Objekt wie einer Ananas im Glas ausstellerischen Gebrauch zu machen. Die Möglichkeiten der Reduktion sind, wie die der Rhetorik, weitaus größer, als auf den ersten Blick zu vermuten ist. Das Beispiel der Ausstellung „Ausgepackt“, die 2007 im Stadtmuseum Erlangen zu sehen war, soll dies illustrieren.

Dort fand sich die Ananas im Glas in einer Installation, die anzeigte, dass sie nur ein Exponat unter vielen ist. Es ging auch dort nicht vordergründig darum, eine Frucht oder eine botanische Sammlung zu repräsentieren, sondern Arbeitstechniken der Wissenschaft auszustellen. Es wurde sichtbar, was auch in Göttingen zu sehen war: dass das Sammeln eine wissenschaftliche Technik ist, die in den verschiedensten Fächern angewandt wird. Der Raum der Ausstellung in Erlangen trug den Titel Depot.

Die Ananas findet sich links neben der Tür im zweiten Regal von oben, inmitten anderer Objekte. Die Dinge in diesem Raum Exponate zu nennen, ist zweifellos ein Grenzfall der Begriffsverwendung. Der Begriff ist dennoch berechtigt.

Das Zusammenspiel von Reduktion und Rhetorik kommt auch hier zum Tragen. Die Reduktion ist überdeutlich: Dies ist kein Depot, sondern ein buntes Sammelsurium aus ganz verschiedenen Depots verschiedener Sammlungen..$^{40}$ Die Rhetorik ist nicht weniger konsequent: Die nicht vorhandenen Objektlegenden und die Gitter betonen das inszenatorische Moment. Zusammen mit der unüberschaubar scheinenden Menge der Objekte bilden sie eine starke rhetorische Geste. Hier ist zum einen auf das Wesentliche reduziert - nämlich plakativ weggelassen - und zum anderen synthetisiert - nämlich aufs Neue gezielt angesammelt - worden. Die Anordnung im Raum wird unweigerlich

39 Vgl. Silke Glitsch: Die Paulinerkirche von der Inauguration bis zum 275-jährigen Jubiläum der Georg-August-Universität Göttingen. In: Dinge des Wissens: Die Sammlungen, Museen und Gärten der Universität Göttingen. Hrsg. von der Georg-August-Universität Göttingen. Göttingen: Wallstein, 2012. S. 117-125.

40 Bilder vom Raum und eine Liste der Objekte finden sich in: Ausgepackt. Die Sammlungen der Universität Erlangen-Nürnberg. 20. Mai bis 29. Juli 2007. Stadtmuseum Erlangen. Dokumentation der Ausstellung. Hrsg. von Udo Andraschke, Thomas Engelhardt und Marion Marie Ruisinger. Erlangen: Stadtmuseum, 2007. S. 46-51. 


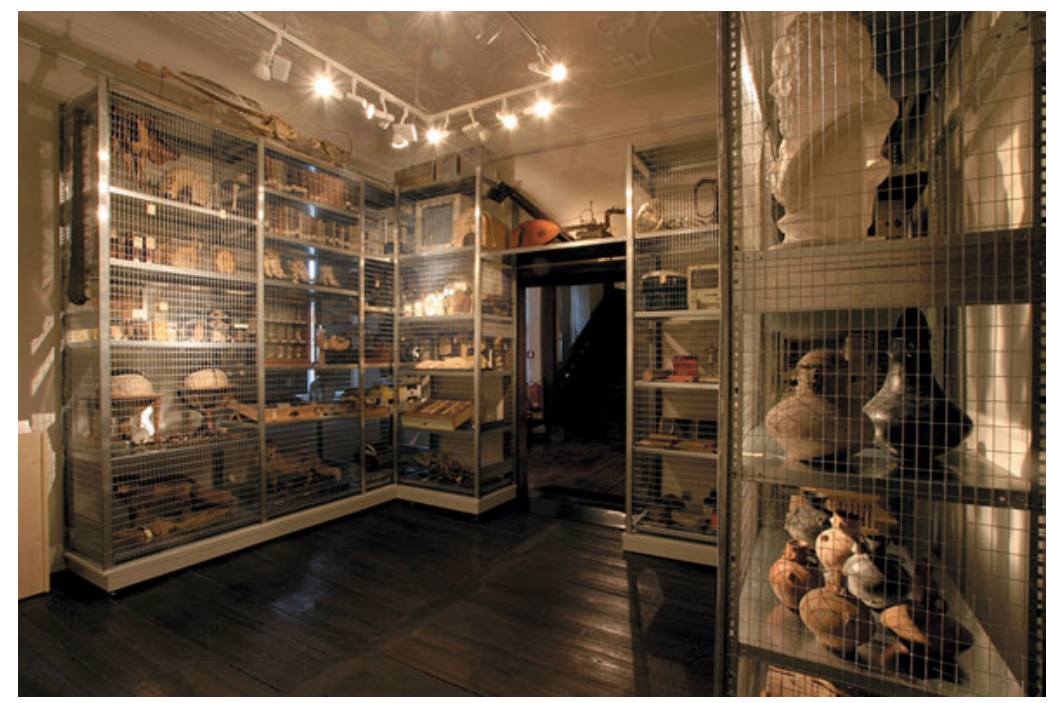

Abb. 12: Ananas in der Erlanger Ausstellung. Ausstellungsraum „Depot“ (Foto: Udo Andraschke).
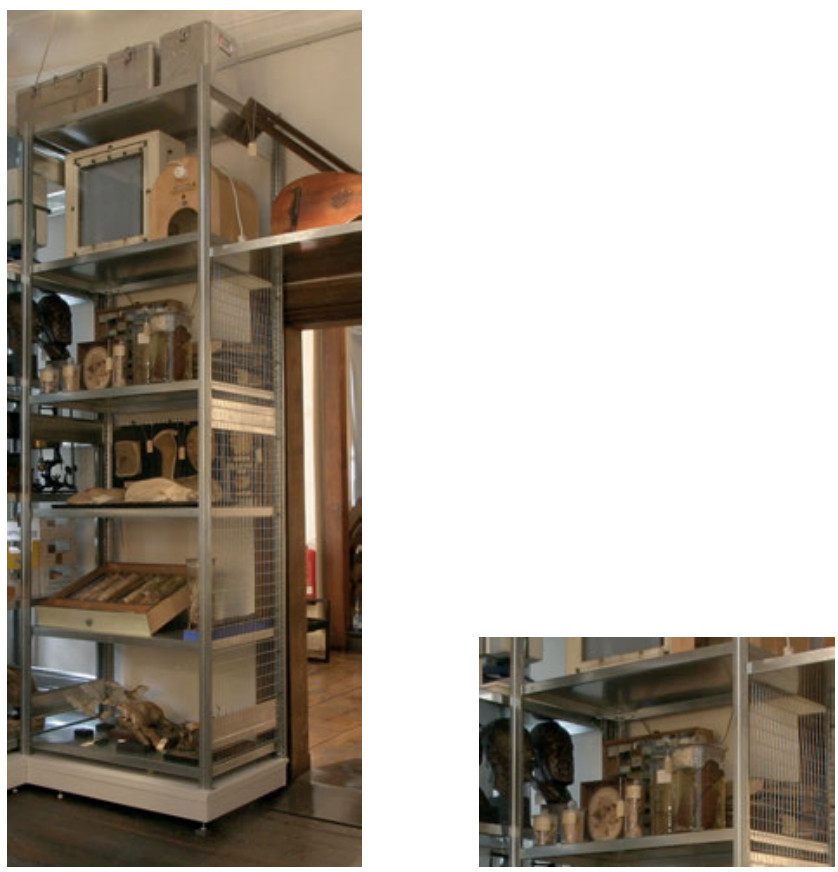

Abb. 13: Ananas in der Erlanger Ausstellung. Ausstellungsraum „Depot“, Details (Fotos: Udo Andraschke). 


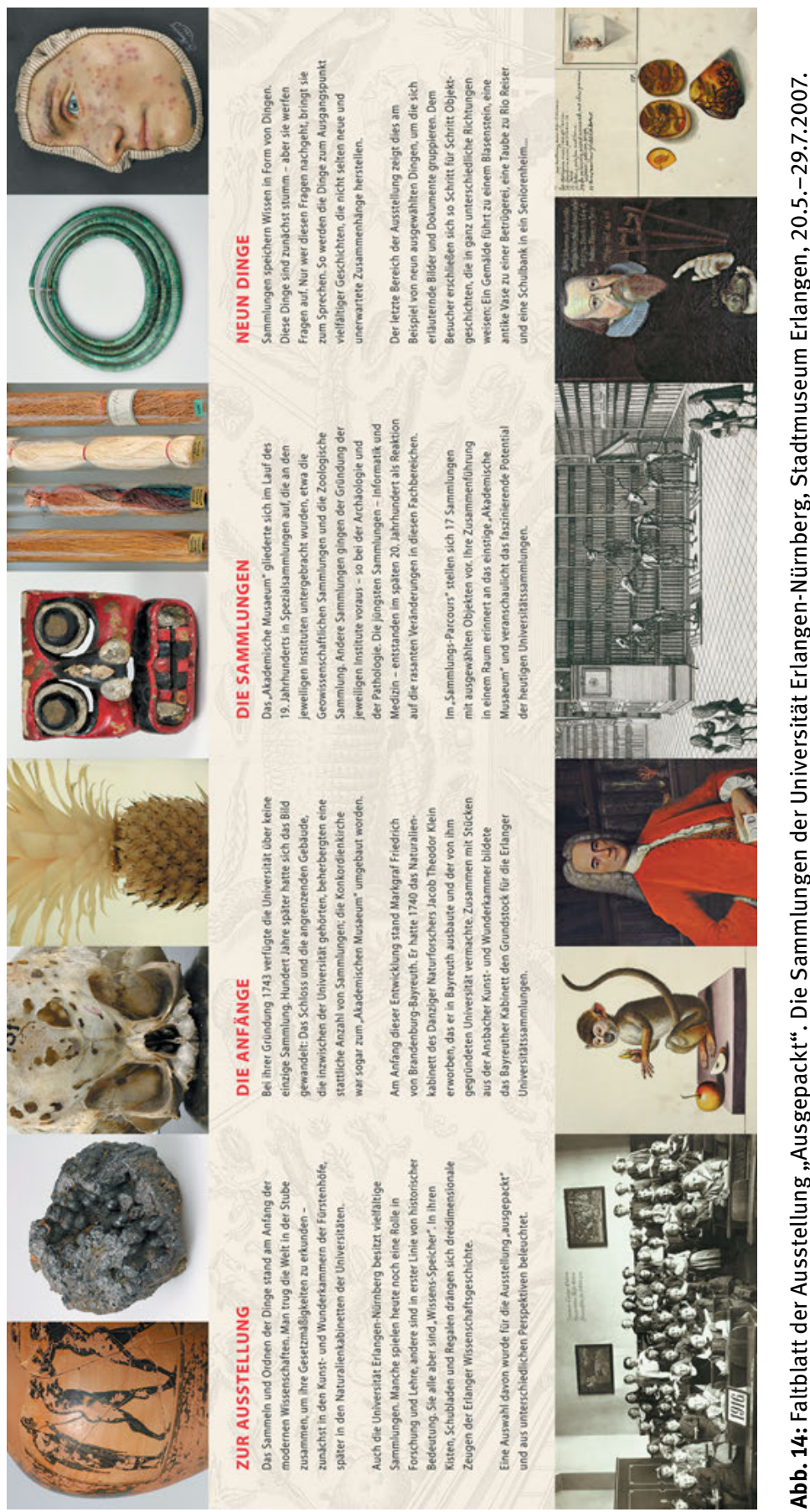


selbstreferentiell, ausgestellt wird eine Organisationsform von Dingen und von Wissen: das Depot. Viele Dinge gemeinsam bilden auf engem Raum den Kontext füreinander. Das Einzelne geht darin nicht unter, sondern auf. Der Kontext, den ein einzelnes Ding mit sich führt, kommt nicht für sich zur Geltung, sondern als Teil eines größeren Ganzen.

Aus der Sicht des Raumes ist die Ananas ein Detail. Im Gesamt der Ausstellung wurde sie - eben als Detail - noch in ein weiteres rhetorisches Spiel einbezogen. Auf dem Faltblatt, mit dem die Ausstellung angekündigt wurde, hatte auch die Ananas einen Platz gefunden.

Der Aufbau des Flyers funktioniert nach dem nun schon bekannten Prinzip pars pro toto. Die rhetorische Aufladung der Exponate wird hier einen feinen Schritt weiter getrieben. Die beiden Reihen von Bildern stehen in einem funktionalen Verhältnis. Die Bilder der oberen Reihe betonen den Dingcharakter der Exponate. Die der unteren Reihe tun das nicht: Es sind Abbildungen zweiter Ordnung, Bilder von Bildern. Es handelt sich auch um Exponate (was der Betrachter vorab nicht wissen kann), ihre Inszenierung betont allerdings das Moment der Kontextualisierung. Zwischen den Bildreihen werden, in Textform, die Intentionen der Ausstellungsmacher offengelegt. Man liest die Texte, um zu erfahren, wofür die Gesamtheit der ausgestellten Dinge steht.

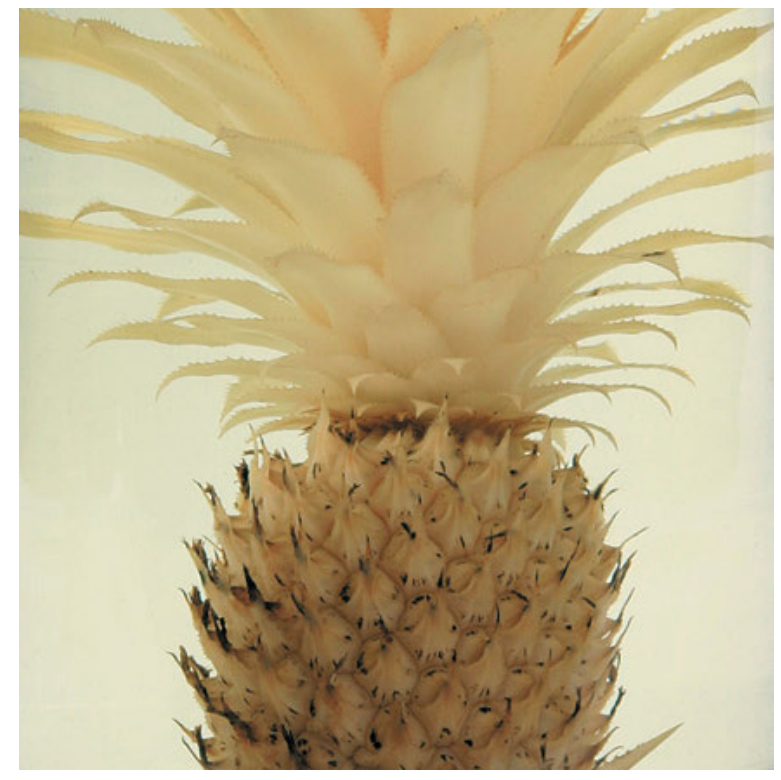

Abb. 15: Ananas, Ausschnitt aus dem Faltblatt der Erlanger Ausstellung „Ausgepackt“ (Foto: Georg Pöhlein). 
Die Abbildung der Ananas auf dem Faltblatt verweist sowohl auf das Exponat als auch auf die Frucht. Es wird allerdings nicht das Präparat gezeigt, sondern nur ein Ausschnitt davon. Der ist photographisch so manipuliert, dass er die Frucht betont. Das Präparat wird im Bild durch die Verfärbung präsentiert. Die Art der Darstellung zeigt an, dass der sichtbare Teil auf etwas verweist, das nicht zu sehen ist. Zu erkennen sind das Ding und seine stellvertretende Funktion. Rezeptionstheoretisch ließe sich von der Erzeugung von Fallhöhe sprechen: die sorgfältige Vorbereitung eines rhetorischen Effekts.

Solche Überlegungen hören auf, trivial zu sein, sobald man ein einzelnes Ding und seine Bedeutung durch die Kontexte hindurch verfolgt. Wer die Ananas in der Ausstellung suchte, wurde enttäuscht. Man stieß auf das Depot und eine Frage, vielleicht sogar mehrere. Die Frage, wie man eine Ananas oder ein Depot ausstellt, ist ein Spezialfall der Frage, wie man Wissen sichtbar macht. Damit haben Museen und Universitäten gleichermaßen zu tun.

Auch die Göttinger Ausstellungsmacher haben versucht, das gemeinhin Unsichtbare sichtbar zu machen und Depots ausgestellt. ${ }^{41} \mathrm{Zu}$ diesem Zweck hat eine Photographin Streifzüge durch die Göttinger Depots unternommen. Ihre Bilder sind auf schwarzen Wänden angebracht worden, die an beiden Längsseiten des Mitteltisches der Ausstellung laufen. Man kann die Bilder von den Dingen aus nicht sehen, sie sind nur von der Außenseite sichtbar.

Der Unterschied zum Depot, das aus Dingen besteht, ist offensichtlich. Der Weg der Sichtbarmachung ist im Vergleich zur Erlanger Ausstellung ein anderer. Zwischen Depot und Ausstellung ist ein weiteres Medium zur Vermittlung benutzt worden, das die Dinge auf ganz andere Art reduziert: Sie sind abwesend. Und dank der räumlichen Inszenierung hüllen sie mit ihrer Abwesenheit die anwesenden Dinge ein. Auch das ist eine rhetorische Geste.

Sie steht der Behauptung der Photographin entgegen, ihre Bilder zeigten „die Magie der Dinge, ihre Aura und Ausstrahlung“..42 Das tun die Bilder in der Ausstellung auch, aber nicht durch das, was sie zeigen, sondern durch den Kontrast zu den Exponaten.

41 Für Pomian steht der Zusammenhang zwischen dem Sichtbaren und dem Unsichtbaren im Zentrum seiner Überlegungen zum Sammeln. Es dürfte lohnend sein, seine Schriften noch einmal auf diesen Aspekt hin zu lesen; vgl. vor allem Pomian: Ursprung.

42 Isi Kunath: Ich will nicht zeigen, ich lasse sehen. In: Dinge des Wissens: Die Sammlungen, Museen und Gärten der Universität Göttingen. Hrsg. von der Georg-August-Universität Göttingen. Göttingen: Wallstein, 2012. S. 192. 


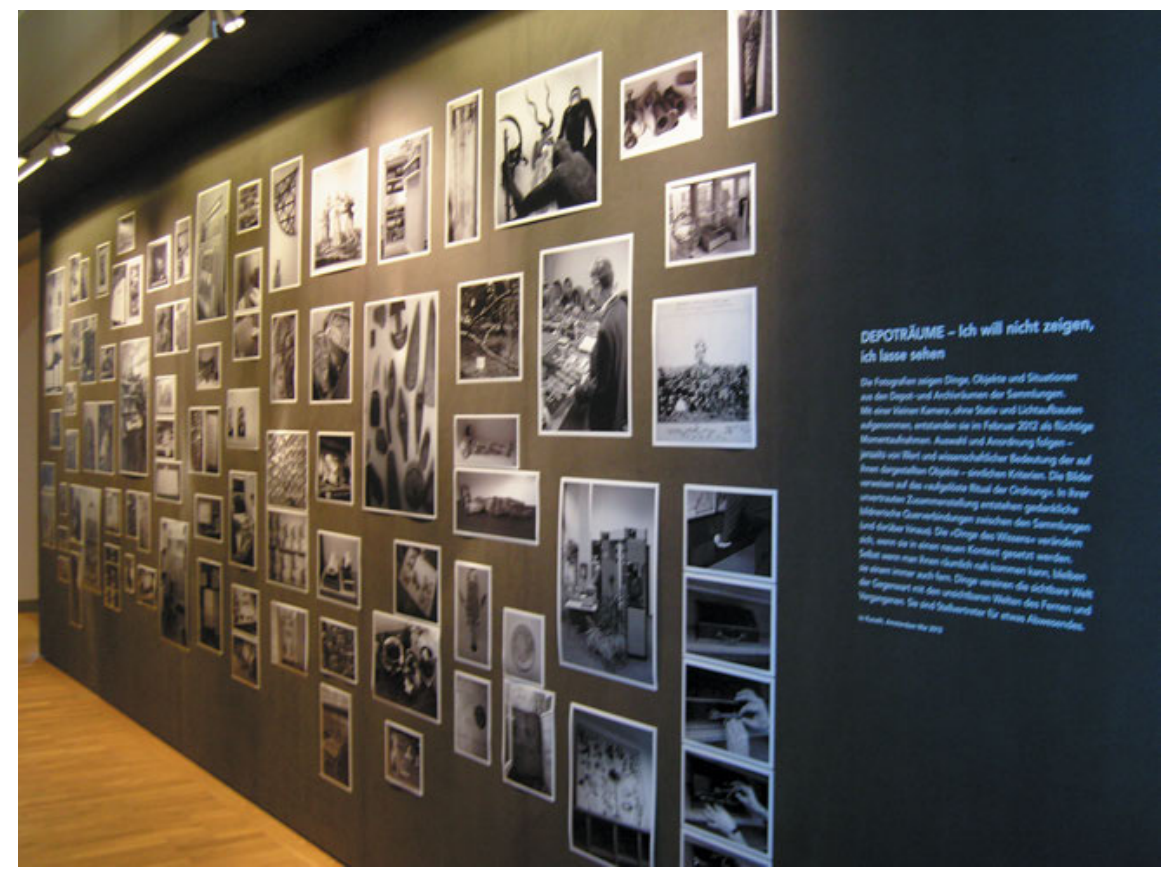

Abb. 16: Abschnitt „Depot“ der Göttinger Ausstellung (Foto: Holger Helbig).

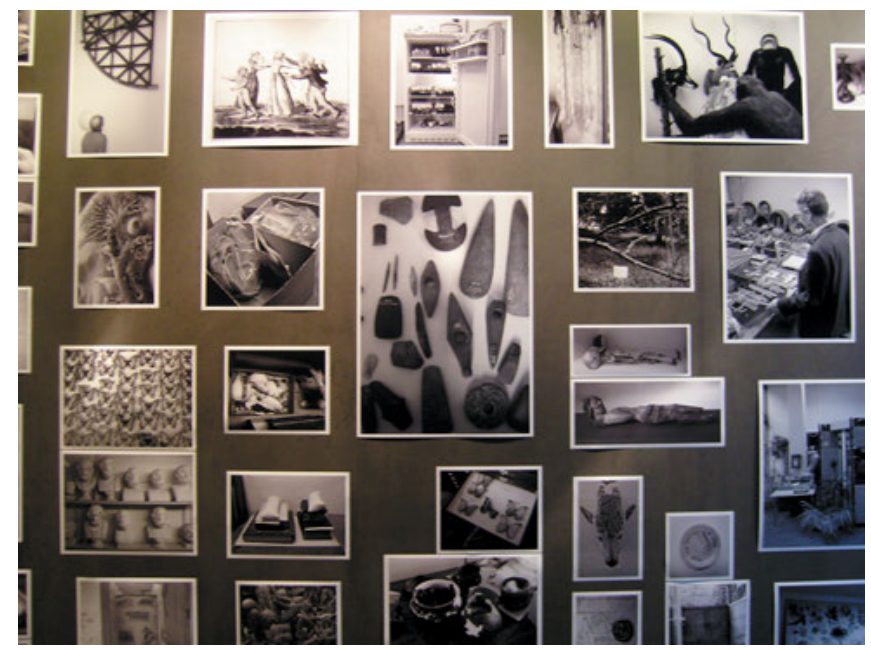

Abb. 17: Abschnitt „Depot“ der Göttinger Ausstellung, Detail (Foto: Holger Helbig). 


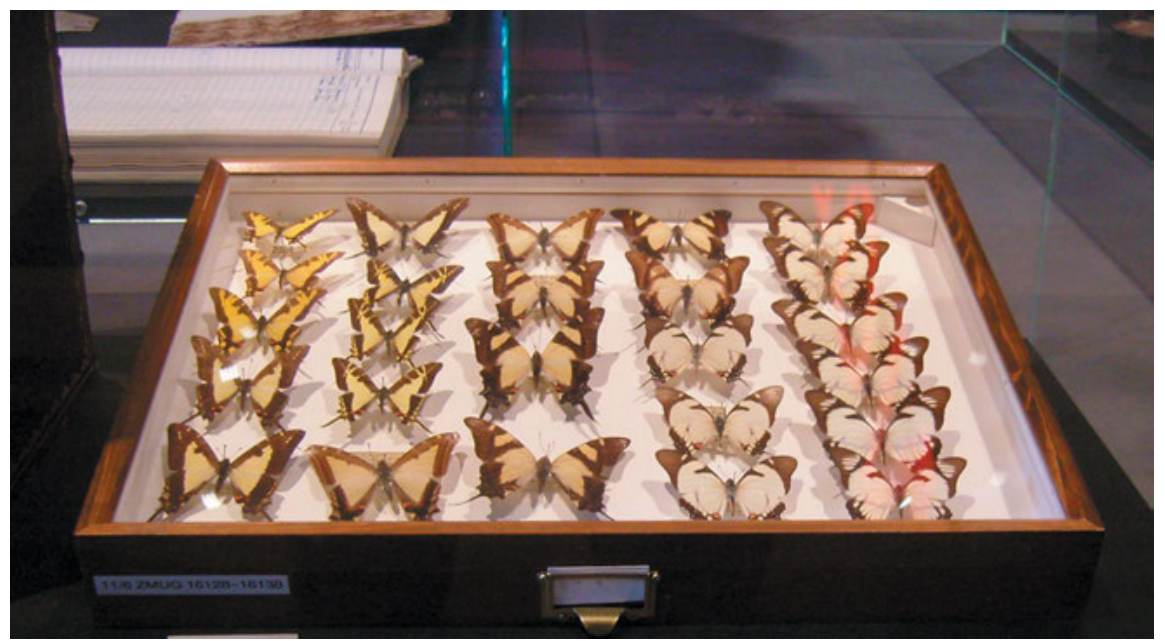

Abb. 18: Das Exponat (Schmetterlinge) (Foto: Holger Helbig).

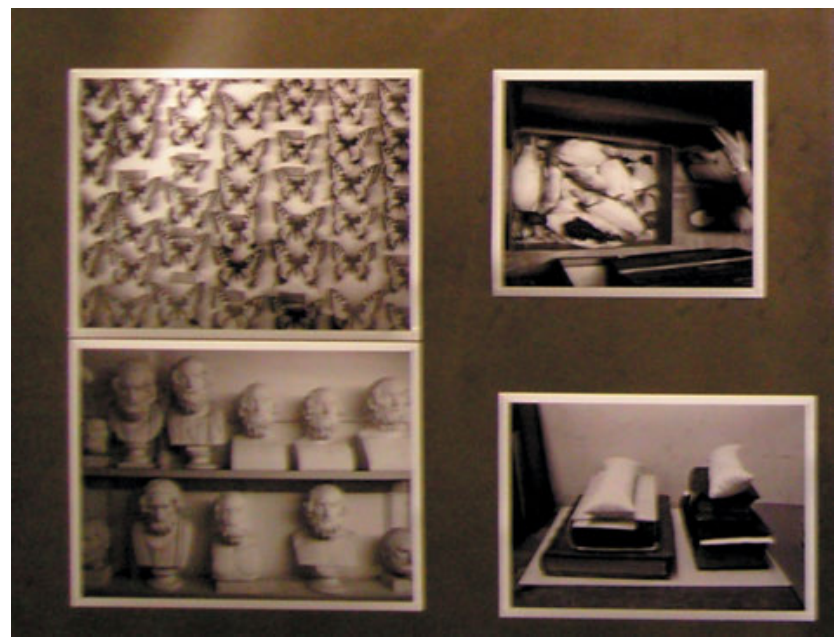

Abb. 19: Das Bild vom Exponat (Schmetterlinge) (Foto: Holger Helbig).

Die Photographien sind eine Ansammlung von Zeichen. In ihrer Gesamtheit machen sie eine Funktion von Sammlungen sichtbar. Und nach dem Abbau der Ausstellung verfügt die Universität Göttingen über eine neue Sammlung: die der Photographien. An beiden Ausstellungen, in Göttingen und Erlangen, war zu erkennen, dass die Rhetorik durch Reduktion erkauft wird. In dem einen Fall, in Erlangen, wurde die inszenatorische Geltung des konkreten Exponats - 
der Ananas - reduziert und damit der Bezug zu den Kontexten weitgehend minimiert. In dem anderen Fall, in Göttingen, wurde der dingliche Charakter der auszustellenden Idee - das Depot als Einheit der Wissensorganisation - nahezu vollständig reduziert. In beiden Fällen fungierte die Ansammlung von Exponaten als ein Zeichen.

Die Sache lässt sich aus verschiedenen Perspektiven betrachten, sowohl aus der Sicht eines einzelnen Dings als auch aus der des sichtbar werdenden Wissens. Aus der ersten Blickrichtung lassen sich Stationen der Bedeutungsveränderung beschreiben, aus der zweiten Formen der Inszenierung. Beide Male wird derselbe Zusammenhang erfasst, nämlich das Zusammenspiel von Reduktion und Rhetorik.

Nach dem Durchgang durch die Beispiele lässt sich genauer sagen, was mit dem Begriffspaar erfasst wird: nicht das Ding, sondern der Umgang damit.

Auf dem Weg in die Ausstellung werden die Zahl der Dinge und beim einzelnen Ding die Kontexte reduziert: ihre Wahrnehmbarkeit, ihre unmittelbare Umgebung. Für die öffentliche Betrachtung wird das einzelne Ding durch Re-Kontextualisierung rhetorisch aufgeladen. Dabei kann der Kontext aus anderen Dingen und/oder aus expliziten Aussagen bestehen. In jedem Fall geht es darum, die Aufmerksamkeit der Betrachter zu steuern und die Wahrnehmung eines Dings mit der Behauptung seiner Bedeutung zu verbinden.

Jochen Hennigs eingangs aufgegriffene Frage nach der „Vereinzelung und Gegenüberstellung von Objekten“ sowie der „Form ihrer Ent- und Neukontextualisierung“ gilt den Verfahren der Bedeutungszuschreibung. Sie lässt sich beantworten, indem man für jeden konkreten Fall das Verhältnis von Reduktion und Rhetorik näher bestimmt.

Eine solche Konkretisierung ließe sich zu einer Ausstellungstheorie entwickeln. Den Grundstein für Überlegungen solcher Art hat Stephen Bann gelegt, der für die implizite Theorie der frühen französischen Geschichtsmuseen den Terminus ,Poetik‘ reklamierte. ${ }^{43}$ Unter Rückgriff auf Michel Foucault und Hayden White rekonstruierte er das Geschichtsverständnis der Museumsgründer mithilfe von Tropen: Museen, in denen das Original einen besonderen Status hat, bezeichnet er als synekdochisch; Ausstellungen, in denen Kopien die Originale vertreten, nannte er metonymisch. In der ersten Kategorie vermittelt das authentische Ding die Illusion des direkten Zugangs zur Quelle. In der zweiten Kategorie fungieren die Kopien als Verweise auf das Abwesende. Zum Ausarbeiten dieser Differenz nutzt Bann einen strukturalistischen Ansatz. Er setzt die Historie als System zum

43 Vgl. zum Folgenden: Stephen Bann: Historical Text and Historical Object: The Poetics of the Musée de Cluny. In: History and Theory 17.3 (1978). S. 251-266. 
Museum als Syntagma ins Verhältnis: Das einzelne Ding wird als ein Term in einer Aussage verstanden, der von dem einen Satz - dem originalen, inzwischen historischen - in einen anderen Satz - den musealen, nun aktuellen - übernommen wird. Im Museum werden Aussagen erzeugt, die die Historie erfassen sollen. Dazu werden verschiedene Sorten von Dingen in verschiedenen Konstellationen angeordnet.

Von deutlich anderen Voraussetzungen ausgehend ist Mieke Bal bei ihren Überlegungen zum Sammeln zu einem frappierend ähnlichen Beschreibungssystem gekommen. Ihr Ausgangspunkt ist narratologisch. Sie fragt sich, auf welche Weise Dinge als vermeintlich objektive Artefakte $\mathrm{zu}$ „,subjektivierte[n] Elemente[n] einer Erzählung“ werden können. ${ }^{44}$ Sie untersucht das Zustandekommen von Sammlungen: Welche Kriterien liegen der Wahl der Objekte und den Ordnungen, in die das Objekt bei der Aufnahme in eine Sammlung eingefügt wird, zugrunde? Auch sie spricht von Metapher, Metonymie und Synekdoche, wenn sie klärt, welche Beziehung zwischen dem ursprünglichen System und dem Syntagma der Sammlung bestehen. Bal greift dabei allerdings auf die Psychoanalyse zurück und entwickelt ihre Kategorien als Varianten der Symbolisierung nach Freud. ${ }^{45}$

Für einen ersten Beschreibungsversuch der betrachteten Beispiele ließe sich - an Bann und Bal anknüpfend und sie vermischend - in etwa sagen: Der Gebrauch der Ananas im Glas in der Göttinger Ausstellung ist stark synekdochisch, insofern die Frucht ein Fach vertritt, in dem das Präparat zur Anwendung kam, und schwach synekdochisch, insofern auf eine Arbeitstechnik verwiesen wird, deren Ergebnis zu sehen ist. Der Verweis auf das Sammeln und Ordnen kommt durch die Konstellation der Exponate zustande, mehrere synekdochische Exponate werden zu einer Aussage integriert: ein Phänomen, das Bann ,organisch nennt.

Beim Gebrauch, der in der Erlanger Ausstellung von dem Feuchtpräparat gemacht wurde, dominiert das metonymische Moment: Der Verweis auf die Botanik geht in der Inszenierung unter. Das Ding vertritt etwas anderes. Die Installation des Depots insgesamt ist metaphorisch. Es wird auf mehrere Analogien gesetzt, man würde beschreibend eher von einer Darstellung als einer Ausstellung sprechen. In der Sprache der Museologen behilft man sich, indem man an dieser Stelle

44 Mieke Bal: Vielsagende Objekte: Das Sammeln aus narrativer Perspektive. In: dies.: Kulturanalyse. Hrsg. von Thomas Fechner-Smarsly und Sonja Neef. Frankfurt a. M.: Suhrkamp, 2002. S. 117-145, hier S. 120. Im Original: Telling Objects: A Narrative Perspective on Collecting. In: The Cultures of Collecting. Hrsg. von John Elsner und Roger Cardinal. London: Reaktion Books, 1994. S. 97-115. Vgl. dazu auch Mieke Bal: Double Exposures: The Practice of Cultural Analysis. Hoboken: Taylor and Francis, 2012.

45 Vgl. Bal: Objekte, S. 132. 
sagt, es werde eine Organisationsform von Wissen exponiert. Der Aufbau dieser Depot-Inszenierung wäre nach Bann ironisch, weil das Bewusstsein der funktionalen Nutzung der Präparate erkennbar ist - oder anders gesagt: weil die Reduktion mit ausgestellt wird.

Dem Exponieren des Depots in Göttingen dagegen fehlt dieses Moment. Es wird nicht ein Präparat zum Exponat. Die Rede von der Aura, die sich medial vermitteln ließe, bleibt eine Behauptung im Syntagma der Ausstellung. Dass ihr kein Phänomen in der Realität des Depots entspricht, konnte man in Erlangen sehen: Das Depot ist eben nicht auf die Sichtbarmachung einzelner Dinge ausgerichtet, sondern auf deren ebenso geordnete wie platzsparende Unterbringung. Diese Konstellation ließe sich in Ermangelung eines anderen Vorschlags gutwillig dem Ornat von Ausstellungen zurechnen. Oder man spricht von einem Kategorienfehler.

Offensichtlich ist man bei der Inszenierung des Depots als Ausstellungsgegenstand in Erlangen und Göttingen von ähnlichen Überlegungen ausgegangen und hat auf dieselben Mechanismen gesetzt, sie aber ganz unterschiedlich zur Darstellung gebracht. Diese Beobachtung spricht für die Annahme, dass das Potential einer an Bann und Bal anknüpfenden Theorie in ihrer rhetorischen Präzisierung liegt. ${ }^{46}$ Es liegt nahe, die beiden Darstellungsweisen hinsichtlich der Kombination von detractio und synthesis auszuarbeiten. Was ist jeweils weggelassen worden, um was auszustellen - und um was zu behaupten? Ein solches terminologisches Ausbuchstabieren des Zusammenhangs von Reduktion und Rhetorik dürfte so reizvoll wie lohnend sein.

Eine Grundlegung dazu hat Gottfried Korff in einer ganzen Reihe von Aufsätzen geschaffen. Er fasst im Anschluss an Bann museale Inszenierung generell als „ästhetisch bewußte Rahmung“ auf, ,als Organisation der Anschauung, mit dem Ziel, die Lesart der Objekte zu befördern, metonymisch, synekdotisch, ironisch“. ${ }^{47}$ Wie solche Lesarten in Ausstellungen inszenatorisch befördert oder behindert werden können, hat Jana Scholze mithilfe eines weiter gefassten semiotischen Ansatzes beschrieben. Ihre Aufmerksamkeit gilt den „typischen Formen der Ausstellungspräsentation“. ${ }^{48}$ In einer Reihe von Einzelanalysen hat sie für ver-

46 Gelegentlich werden die Überlegungen von Bann und Bal in der einschlägigen Literatur aufgegriffen. Eine taugliche Theorie von Ausstellungen allerdings scheint nicht in Sicht.

47 Gottfried Korff: Speicher und/oder Generator: Zum Verhältnis von Deponieren und Exponieren im Museum. In: Museumsdinge deponieren - exponieren. Hrsg. von Martina Eberspächer, Gudrun Marlene König und Bernhard Tschofen. Köln: Böhlau, 2002. S.167-178, hier S. 173. Herv. im Orig.

48 Jana Scholze: Medium Ausstellung: Lektüren musealer Gestaltungen in Oxford, Leipzig, Amsterdam und Berlin. Bielefeld: transcript, 2004. S.12. 
schiedene Ausstellungen das Zustandekommen von Bedeutung nachvollzogen, indem sie die konkreten Ausstellungskontexte hinsichtlich der kulturellen Codes und deren Hierarchie untersuchte. ${ }^{49}$ Auf ein solches Vorgehen könnte eine rhetorisch ausgerichtete Theorie zurückgreifen. Es ließe sich dabei auch beobachten, welche Möglichkeiten sich bieten, um Fachwissen und seine Entwicklung aus der Universität hinaus einer größeren Öffentlichkeit darzustellen. Dass es dabei um besondere Formen der Reduktion geht, und um eine Rhetorik, die auf Anschaulichkeit und Verständlichkeit ausgerichtet ist, kann man in gut gemachten Ausstellungen - und im Actionkino - sehen.

\section{Literatur}

Andraschke, Udo, Thomas Engelhardt und Marion Marie Ruisinger (Hrsg.): Ausgepackt. Die Sammlungen der Universität Erlangen-Nürnberg. 20. Mai bis 29. Juli 2007. Stadtmuseum Erlangen. Dokumentation der Ausstellung. Erlangen: Stadtmuseum, 2007.

Bal, Mieke: Double Exposures. The Subject of Cultural Analysis. New York u. a.: Routledge, 1996.

Bal, Mieke: Exposing the Public. In: A Companion to Museum Studies. Hrsg. von Sharon Macdonald. Malden, MA: Wiley-Blackwell, 2011. S. 525-542.

Bal, Mieke: Telling Objects. A Narrative Perspective on Collecting. In: The Cultures of Collecting. Hrsg. von John Elsner und Roger Cardinal. London: Reaktion Books, 1994. S. 97-115.

Bal, Mieke: Vielsagende Objekte. Das Sammeln aus narrativer Perspektive. In: dies.: Kulturanalyse. Hrsg. von Thomas Fechner-Smarsly und Sonja Neef. Frankfurt a. M.: Suhrkamp, 2002. S. 117-145.

Bann, Stephen: Historical Text and Historical Object. The Poetics of the Musée de Cluny. In: History and Theory 17 (1978). S. 251-266.

Bencard, Adam, und Thomas Söderqvist: Do things talk? In: The Exhibition as Product and Generator of Scholarship. Hrsg. von Susanne Lehmann-Brauns, Christian Sichau und Helmut Tischler. Berlin: Max-Planck-Institut für Wissenschaftsgeschichte, 2010 [Preprint, Bd. 399]. S. 93-102.

Bräunlein, Peter J.: Material Turn. In: Dinge des Wissens. Die Sammlungen, Museen und Gärten der Universität Göttingen. Hrsg. von der Georg-August-Universität Göttingen. Göttingen: Wallstein, 2012. S. 30-44.

Brüning, Jochen: Wissenschaft und Sammlung. In: Bild, Schrift, Zahl. Hrsg. von Sybille Krämer und Horst Bredekamp. München: Fink, 2003. S. 87-113.

Bundesministerium für Bildung und Forschung (Hrsg.): Förderrichtlinien: „Die Sprache der Objekte - Materielle Kultur im Kontext gesellschaftlicher Entwicklungen“vom 28. März 2012. Amtlicher Teil des Bundesanzeigers (BAnz AT). 11. April 2012.

49 Zur Darstellung ihres Selbstverständnisses vgl. Jana Scholze: Kultursemiotik: Zeichenlesen in Ausstellungen. In: Museumsanalyse. Methoden und Konturen eines neuen Forschungsfeldes. Hrsg. von Joachim Baur. Bielefeld: transcript, 2010. S. 121-148, hier bes. S. 137-145. 
Clever, Joanne: Doing Children's Museums. Charlotte, VT: Williamson Publishing, 1992.

Collins, Julius L.: The Pineapple. Botany, Cultivation, and Utilization. London: Hill, 1960.

Daston, Lorraine: Speechless. In: Things That Talk. Object Lessons from Art and Science. Hrsg. von Lorrain Daston. New York: Zone Books, 2008. S. 9-24.

Evelyn, John: The Diary. Hrsg. von E. S. de Beer. London: Oxford University Press, 1959.

Franke, Gunther (Hrsg.): Nutzpflanzen der Tropen und Subtropen. Bd. 2. Stuttgart: Ulmer, 1994.

Franke, Wolfgang: Nutzpflanzenkunde. Nutzbare Gewächse der gemäßigten Breiten, Subtropen und Tropen. 4. Auflage. Stuttgart: Thieme, 1989.

Geimer, Peter: Über Reste. In: Dingwelten. Das Museum als Erkenntnisort. Hrsg. von Anke te Heesen und Petra Lutz. Köln: Böhlau, 2005. S. 109-118.

Georg-August-Universität Göttingen (Hrsg.): Dinge des Wissens. Flyer zur Ausstellung zum 275. Jubiläum der Georg-August-Universität Göttingen. Göttingen: ohne Verlag, 2012.

Genaust, Helmut: Etymologisches Wörterbuch der botanischen Pflanzennamen. 3. Auflage. Basel: Birkhäuser, 1996.

Glitsch, Silke: Die Paulinerkirche von der Inauguration bis zum 275-jährigen Jubiläum der Georg-August-Universität Göttingen. In: Dinge des Wissens. Die Sammlungen, Museen und Gärten der Universität Göttingen. Hrsg. von der Georg-August-Universität Göttingen. Göttingen: Wallstein, 2012. S. 117-125.

Heesen, Anke te: Theorien des Museums. Hamburg: Junius, 2012.

Hennig, Jochen: Wissensdinge - Wissensanordnungen - Wissensorte. Zum Ausstellen von Universitätssammlungen. In: Dinge des Wissens. Die Sammlungen, Museen und Gärten der Universität Göttingen. Hrsg. von der Georg-August-Universität Göttingen. Göttingen: Wallstein, 2012. S. 20-29.

Knight, Thomas Andrew: Das Ganze der Ananaszucht, oder die verschiedenen Arten, wie man Ananas gezogen hat und noch zieht, von der ersten Einführung dieser Frucht in Europa bis zu den neuesten Kulturverbesserungen. Weimar: Voigt, 1881.

Korff, Gottfried: Betörung durch Reflexion. Sechs um Exkurse ergänzte Bemerkungen zur epistemischen Anordnung von Dingen. In: Dingwelten. Das Museum als Erkenntnisort. Hrsg. von Anke te Heesen und Petra Lutz. Köln: Böhlau, 2005. S. 89-107.

Korff, Gottfried: Zur Eigenart der Museumsdinge [1992]. In: ders.: Museumsdinge. deponieren - exponieren. Köln: Böhlau, 2002. S.140-145.

Korff, Gottfried: Speicher und/oder Generator. Zum Verhältnis von Deponieren und Exponieren im Museum. In: Museumsdinge. deponieren - exponieren. Hrsg. von Martina Eberspächer, Gudrun Marlene König und Bernhard Tschofen. 2. erg. Auflage. Köln: Böhlau, 2007. S. 167-179.

Kunath, Isi: Ich will nicht zeigen, ich lasse sehen. In: Dinge des Wissens. Die Sammlungen, Museen und Gärten der Universität Göttingen, Hrsg. von der Georg-August-Universität Göttingen. Göttingen: Wallstein, 2012. S. 192.

Locke, John: An Essay Concerning Human Understanding. Hrsg. von Roger Woolhouse. London: Penguin Books, 1997.

Martyr von Anghiera, Petrus: Acht Dekaden über die neue Welt. Bd. 1. Darmstadt: Wissenschaftliche Buchgesellschaft, 1972.

Pomian, Krzysztof: Der Ursprung des Museums. Vom Sammeln. Berlin: Wagenbach, 1988.

Pomian, Krzysztof: Sammlungen. Eine historische Typologie. In: Macrocosmos in Microcosmos. Die Welt in der Stube. Zur Geschichte des Sammelns 1450 bis 1800. Hrsg. von Andreas Grote. Opladen: Leske + Budrich, 1994. S.107-126. 
Rheinberger, Hans-Jörg: Objekt und Repräsentation. In: Mit dem Auge denken. Strategien der Sichtbarmachung in wissenschaftlichen und virtuellen Welten. Hrsg. von Bettina Heintz und Jörg Huber. Zürich: Voldemeer, 2001. S. 55-61.

Scholze, Jana: Medium Ausstellung. Lektüren musealer Gestaltungen in Oxford, Leipzig, Amsterdam und Berlin. Bielefeld: transcript, 2004.

Scholze, Jana: Kultursemiotik. Zeichenlesen in Ausstellungen. In: Museumsanalyse. Methoden und Konturen eines neuen Forschungsfeldes. Hrsg. von Joachim Baur. Bielefeld: transcript, 2010. S. 121-148.

Schwerdtfeger, Michael: Die Ananas im Königreich Hannover und die Sammlung exotischer Pflanzen im botanischen Garten. In: Dinge des Wissens. Die Sammlungen, Museen und Gärten der Universität Göttingen. Hrsg. von der Georg-August-Universität Göttingen. Göttingen: Wallstein, 2012. S. 172-174.

Söderqvist, Thomas: Do things really talk? Are there really hidden stories in objects? Can you write the biography of an artefact? In: Hidden Stories. What do medical objects tell and how can we make them speak? Abstract-Book zur gleichnamigen 16th Biennial EAMHMS Conference vom 13. bis zum 15. September 2012 am Medizinhistorischen Museum der Charité, Berlin. S. 5-12.

Solereder, Hans: Systematische Anatomie der Dicoyledonen. Stuttgart: Enke, 1899.

Thieß, Stephan: ohne Titel. [o. T.]. www.unikik.uni-hannover.de/downloads/mathematik_ diskret.pdf. Skript zum Kurs Diskrete Mathematik der Gauß AG der Leibniz Universität Hannover, Wintersemester 2005/2006. (11. März 2011).

Vogel, Fritz Franz: Das Handbuch der Exponatik. Vom Ausstellen und Zeigen. Köln: Böhlau, 2012.

Volkamer, Johann Christoph: Continuation der Nürnbergischen Hesperidum. Oder: Fernere gründliche Beschreibung der Edlen Zitronat-Zitronen und Pomeranzen-Früchte [...]. Nürnberg: Selbstverl., 1714.

Weil, Stephen E.: From Being About Something to Being for Somebody. In: ders.: Making Museums Matter. Washington, DC: Smithsonian, 2002. S. 28-52.

Wortley Montagu, Lady Mary: The Complete Letters. Bd.1. Hrsg. von Robert Halsband. Oxford: Clarendon Press, 1965.

\section{Abbildungsnachweis}

Abb. 3 und 6 sind entnommen aus: Michael Schwerdtfeger: Die Ananas im Königreich Hannover und die Sammlung exotischer Pflanzen im botanischen Garten. In: Dinge des Wissens: Die Sammlungen, Museen und Gärten der Universität Göttingen. Hrsg. von der Georg-AugustUniversität Göttingen. Göttingen: Wallstein, 2012. S.172-174, hier S. 172.

Abb. 7 ist entnommen aus: Holger Helbig: Ananas. Objektgeschichte. In: Die Sammlungen der Universität Erlangen-Nürnberg. Begleitband zur Ausstellung „Ausgepackt. Die Sammlungen der Universität Erlangen-Nürnberg“, Stadtmuseum Erlangen. Hrsg. von Udo Andraschke und Marion Maria Ruisinger. Erlangen: Friedrich-Alexander-Universität ErlangenNürnberg, 2007, S. 235.

Abb. 14 ist das Faltblatt der Ausstellung „Ausgepackt. Die Sammlungen der Universität Erlangen-Nürnberg, Stadtmuseum Erlangen“, 20.5.-29.7.2007. @ Stadtmuseum Erlangen und Sammlungen der Universität Erlangen-Nürnberg. 